\title{
A Survey and Future Directions on Clustering: From WSNs to IoT and Modern Networking Paradigms
}

\author{
Amin Shahraki, Amir Taherkordi, Øystein Haugen, and Frank Eliassen
}

\begin{abstract}
Many Internet of Things (IoT) networks are created as an overlay over traditional ad-hoc networks such as Zigbee. Moreover, IoT networks can resemble ad-hoc networks over networks that support device-to-device (D2D) communication, e.g., D2D-enabled cellular networks and WiFi-Direct. In these ad-hoc types of IoT networks, efficient topology management is a crucial requirement, and in particular in massive scale deployments. Traditionally, clustering has been recognized as a common approach for topology management in ad-hoc networks, e.g., in Wireless Sensor Networks (WSNs). Topology management in WSNs and ad-hoc IoT networks has many design commonalities as both need to transfer data to the destination hop by hop. Thus, WSN clustering techniques can presumably be applied for topology management in ad-hoc IoT networks. This requires a comprehensive study on WSN clustering techniques and investigating their applicability to ad-hoc IoT networks. In this paper, we conduct a survey of this field based on the objectives for clustering, such as reducing energy consumption and load balancing, as well as the network properties relevant for efficient clustering in IoT, such as network heterogeneity and mobility. Beyond that, we investigate the advantages and challenges of clustering when IoT is integrated with modern computing and communication technologies such as Blockchain, Fog/Edge computing, and 5G. This survey provides useful insights into research on IoT clustering, allows broader understanding of its design challenges for IoT networks, and sheds light on its future applications in modern technologies integrated with IoT.
\end{abstract}

Index Terms-IoT, Clustering, WSNs, Survey, 5G, SDN, Edge, Fog, Blockchain, NFV

\section{INTRODUCTION}

Smart devices have facilitated the pervasive presence of various things, interacting and cooperating with each other through unique addressing schemes-Internet of Things (IoT). IoT, introduced first in 2008-2009 [1], connects billions of devices around the world on top of different network infrastructures, mainly the Internet. IoT aims to integrate different traditional and next-generation network technologies to work simultaneously in a common infrastructure and support different ubiquitous applications [2]. Compared to other networks such as WSNs, IoT nodes are highly heterogeneous [3] thanks to their broad usage in divers application domains and being a key component of Cyber-Physical Systems (CPS) [4].

Many IoT networks resemble ad-hoc networks, following the same pattern of data transmission to the Internet as

Amin Shahraki, Amir Taherkordi and Frank Eliassen are with the Department of Informatics, University of Oslo, Norway

Amin Shahraki and Øystein Haugen are with Faculty of Computer Sciences, $\emptyset$ stfold University College, Halden, Norway
WSNs, e.g., Internet of Vehicles (IoV). In such networks, IoT nodes communicate with each other as an overlay network on top of an existing ad-hoc network protocol, e.g., Zigbee. The basic building blocks of these types of IoT networks are often WSNs in which sensors, along with multitude of everyday objects, communicate, interact and share data on a massive scale [5]. In addition, thanks to the possibility of device-to-device (D2D) communication in networks such as cellular networks, WiFi and Bluetooth, the IoT nodes in such networks can communicate in an ad-hoc manner [6]. This will allow more efficient interaction with co-located IoT nodes and reducing the network overhead. Moreover, there are many IoT applications that run over ad-hoc and MANET networks, such as healthcare [7], smart cities [5], [8], vehicular networks [9], military applications [10], and smart agriculture [11].

In ad-hoc IoT networks, topology management is a critical requirement for efficient and scalable management of the network, as well as the applications deployed over such networks. In ad-hoc networks like WSNs, clustering has been introduced as the most popular approach for topology management. Clustering techniques divide the network to groups of nodes and distribute network functions among the group members to improve efficiency in, e.g., collecting and forwarding data, resource management, and supporting QoS. Many clustering techniques have been proposed for WSN topology management, such as LEACH [12], HEED [13] and TEEN [14], to name a few.

Like WSNs, ad-hoc IoT networks can use clustering for topology management to meet the above performance needs, as well as IoT-specific challenges, e.g., scalability of the network [1]. Sharing many fundamental characteristics with WSNs implies that ad-hoc IoT networks can potentially utilize WSN clustering techniques without having to design them from scratch. However, clustering in IoT can be challenging due to high heterogeneity and mobility of IoT nodes, and integration of IoT with recent computing and networking paradigms such as Edge computing and 5G networks. Therefore, conducting a comprehensive study on existing WSN clustering techniques and investigating their applicability to IoT networks would be significantly advantageous for the IoT research community. Such a survey work can be further completed with reviewing clustering techniques that are already being used in IoT networks. In conducting such an extensive survey, it is crucial to define clear goals on what aspects of the existing techniques should be investigated, and how the scope of applicability to IoT should be envisioned. Considering the 
performance needs of ad-hoc IoT networks, the objectives of clustering, such as reducing energy consumption, load balancing, improving connectivity, etc. should be central to such a survey.

Existing surveys. Most existing clustering surveys study and compare WSN clustering techniques and efficiency of them [15], [16], [17], [18], not comparing the objectives of those techniques and more importantly their applicability in IoT [19]. Most of them cover only main clustering techniques, such as LEACH [12], HEED [13] and FLOC [20], proposed in different forms and extensions. Some other surveys consider only one parameter in designing clustering techniques or network infrastructures like unequal clusters [18]. Another category of surveys covers the techniques derived from the above main techniques to improve clustering performance or support application-specific clustering requirements. For example, in [21] the authors indicate that there are more than 60 extended versions of the LEACH protocol in the literature. Besides, in some survey papers, e.g., [22], clustering has been studied from the viewpoint of reducing energy consumption, while it is not the sole objective of clustering.

This survey work is distinguished from the others by focusing on applicability of clustering in IoT networks and feasibility of migrating existing WSN clustering techniques to IoT networks. The main contributions of this paper include:

- reviewing existing clustering techniques in WSNs from the objectives viewpoint and providing an insightful statistical analysis (cf. Section III);

- investigating applicability of relevant WSN clustering techniques to IoT networks with respect to network properties and identified objectives (cf. Section V);

- for each clustering objective: studying existing IoT clustering solutions and discussing the associated challenges in using clustering in IoT (cf. Section V);

- investigating the advantages of clustering when IoT is integrated with modern computing technologies and paradigms (i.e., Blockchain, Fog and Edge, Software-Defined Networking and Network Function Virtualization, and 5G), as well as exploring the challenges in applying clustering techniques in these types of integration (cf. Section VI).

Review methodology. The methodology adopted for conducting this survey consists of the following steps. First, we extracted the list of main WSN clustering techniques from the relevant papers in reputable conferences and journals, such as ICCCN, WCNC, GLOBCOMM, ICPS, CNCS, SECON, IPDPS, ICDCS, INFOCOM, EWSN, PerCom, SenSys, ITPDS, IEEE IoTJ, ITWC, ITN, ITVT, and ATSN. Based on the extracted relevant papers, we then checked their references and related work to find any other papers that were concealed. Having the main clustering techniques compiled, we finally searched for all other research works that either cited the main techniques like LEACH or proposed their own clustering technique. For that, we read and refined about 500 papers. Finally, over 250 papers were extracted as the distinguished clustering techniques proposed for WSNs and IoT. We filtered the papers based on their quality, publication channel, and number of citations.

\section{Clustering: Basic Concepts And TAXonomy}

Topology management is one of the main challenges in establishing networks, especially in ad-hoc networks [23]. Clustering, as a type of topology management technique, improves the efficiency by dividing the network to groups of nodes and distributing network functions among the group members, e.g., collecting and forwarding data, and resource management. Various network types have utilized clustering for topology management, such as MANET [24], VANET [25], WSN [15], and IoT [26]. From the application layer viewpoint, clustering techniques have been introduced for different types of needs such as resource allocation [27], applying reputation models [28], service discovery [29], intrusion detection [30], fault monitoring [31], and anomaly detection [32]. A cluster is composed of a number of nodes (i.e., members) and has one or more Cluster Heads $(\mathrm{CH})$ to manage the members and shared resources. Moreover, $\mathrm{CHs}$ can collect, fuse, and process members' data, and transfer it to gateway(s). Each network can have one or more gateways (also known as base stations or sinks) that connect CHs to outside of the network. Clustering can address several conventional quality-related objectives, such as reducing resource consumption, improving load balancing and QoS, and fault tolerance. We discuss those objectives in detail in Section V.

\section{A. Clustering Structural Models}

Two primary aspects of clustering include grouping nodes and allocating responsibilities. Grouping methods are generally based on the structure of Voronoi diagrams, but also can be non-Voronoi like chain or spectrum structures. In the Voronoi structure, a 2D or 3D network environment is divided into several unequal sections, called clusters. Each cluster possesses some nodes and possibly interacts with other clusters or gateways directly or through neighbor clusters. In the chain structure, nodes in a cluster connect to each other to reach the corresponding $\mathrm{CH}$. Each node has only two connections with neighbors in the chain to reach the $\mathrm{CH}$ : one outgoing connection to the next hop, and one incoming connection from another node to the current node as the next hop. In the spectrum structure, angles of nodes to base stations (BSs) are as important as the distance to BS for cluster establishment. Node angles are generally captured by the Scanning Sweep method [33]. In both spectrum and chain structures, layering of the network can also be performed to enable multi-hop data transmission and improve the efficiency of the network, especially in terms of resource consumption. Figure 1 depicts the above three clustering structures.

Cluster establishment methods: There are generally two methods to establish clusters in a network: i) Determining clusters by grouping nodes and then selecting one or more nodes as $\mathrm{CH}(\mathrm{s})$, and ii) Selecting CHs first and then inviting other nodes to join a neighbor $\mathrm{CH}$. Joining of nodes to a cluster is mainly based on the physical proximity of nodes and other parameters such as cluster size, number of nodes, and balancing network load and resource consumption. In addition, high-level parameters can be defined as clustering criteria, such as application-specific requirements, local data 


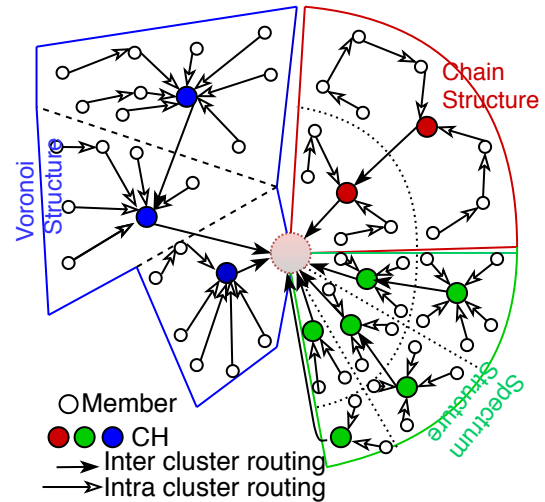

Fig. 1. Different structures of clustering techniques

processing needs (e.g., data fusion and compression), and resource sharing. With respect to $\mathrm{CH}$ selection, there are various techniques as described below:

- In some clustering techniques, resource-rich nodes are predetermined as fixed $\mathrm{CHs}$ for the whole network life time [34]. In networks with homogeneous or resourceconstrained nodes, this method is not efficient. Even in a heterogeneous network, being $\mathrm{CH}$ for a long period of time will drain the node power quickly, leading to node death. Moreover, in case of fixed $\mathrm{CHs}$, mobile nodes and dynamicity of the network can unbalance the number of members and/or cluster load, causing network congestion and inefficient resource consumption.

- In some clustering techniques, randomness is the solution to circulate the $\mathrm{CH}$ responsibility among nodes [12]. Although this is beneficial in homogeneous networks, accidental unfair randomness can lead to network congestion and chronic energy consumption in some CHs.

- The most common solution for such issues is the conscious $\mathrm{CH}$ selection method in which $\mathrm{CHs}$ are selected based on the circumstances of the nodes and the network [35], [36]. In this method, appropriate nodes are actively selected as CHs based on parameters like available resources, location, and number of neighbors.

The $\mathrm{CH}$ selection process can be performed either in a centralized or distributed way. In the centralized model, $\mathrm{CH}$ selection parameters are gathered, compared, analyzed and processed in a central node (i.e., generally BS). Although in this model network-wide comparison of parameters can be performed, it often imposes high overhead due to transmitting many management packets, in particular in large and/or highly dynamic networks with the $\mathrm{CH}$ re-selection capability. On the other hand, distributed methods impose less overhead, but due to relying on local $\mathrm{CH}$ selection criteria, selected $\mathrm{CHs}$ cannot always fulfill the whole network requirements. Due to this fact, distributed methods can cause network inefficiency in different performance aspects, e.g., QoS and load balancing.

Re-clustering methods: Re-clustering refers to any action related to re-selecting $\mathrm{CHs}$ or re-organizing existing clusters. $\mathrm{CH}$ selection methods are mostly designed to react against any unforeseeable circumstances by re-selecting or replacing $\mathrm{CHs}$ dynamically with more appropriate nodes. Moreover, not every node can serve as $\mathrm{CH}$ for a long period of time because of resource depletion, thereby the $\mathrm{CH}$ role should be rotated among appropriate nodes during the network lifetime. With respect to re-organization of clusters, events like network congestion, dead nodes, and unbalanced network load can also trigger re-clustering. There are two methods to trigger re-clustering: i) Time-based method: The network will be re-clustered at a certain time to balance resource consumption among nodes. This method is generally used in homogeneous networks with predetermined network load, and ii) Event-based method: An event triggers part of or the whole network to re-select $\mathrm{CHs}$ and possibly re-cluster, e.g., exceeding resource usage thresholds like energy, CPU, bandwidth consumption, or high resource consumption in a certain time. The combination of time-based and event-based methods can be used for re-clustering as well. Depending on which re-clustering conditions hold first, the corresponding method will be triggered.

Data forwarding in clustering techniques: The network data can be transferred in its raw format or as a fused value (i.e., data fusion). In clustered networks, $\mathrm{CHs}$ are used to gather and fuse the members' data. The $\mathrm{CH}$ can transmit individual data items to BS or send the fused values. Since common clustering techniques are based on the proximity of the nodes providing data for the same application, it would be possible to perform data aggregation in $\mathrm{CHs}$ and reduce the amount of data to be transmitted. In addition, in some cases, CHs are able to compress data to reduce the data volume. Last but not least, data can be processed locally in $\mathrm{CHs}$ and results can be sent to the gateway(s). There are two methods to transmit packets from $\mathrm{CHs}$ to $\mathrm{BS}(\mathrm{s})$ :

- CHs can send data directly to BS. In the direct communication, each $\mathrm{CH}$ transmits data directly to $\mathrm{BS}(\mathrm{s})$ causing energy depletion in case of a long distance $(d)$ based on Eq.1 [37].

$$
E_{\text {transmit }}=F\left(d^{2}\right)
$$

- CHs can use middle nodes (often other $\mathrm{CHs}$ ) to forward data to the gateway(s), called inter-cluster routing. Although it reduces energy consumption, it increases delay. This method also needs establishing efficient routes from $\mathrm{CHs}$ to the gateway(s) causing network overhead.

Besides the communication between $\mathrm{CHs}$ and $\mathrm{BS}(\mathrm{s})$, nodes in a cluster can connect to their $\mathrm{CHs}$ directly or indirectly, called intra-cluster routing. However, it is used in special cases e.g., blind spaces or big-sized clusters. This routing method can also impact QoS, e.g., increasing delay [37], [38].

\section{Clustering in WSNS}

WSNs are a network of devices, denoted as nodes, which can sense the environment and transmit the sensed data gathered from the monitored field wirelessly. The sensed data is transmitted, possibly via multiple hops, to a BS node that can process it locally or forward it through a gateway to other networks, e.g., the Internet. There exist different types of WSNs, such as Terrestrial WSNs [39], Underground WSNs [40], Underwater WSNs [41], Multimedia WSNs [42], and Mobile WSNs and Wireless Sensor and Actuator Networks (WSANs) [43]. 
WSNs are often considered as infrastructure-less resourceconstrained networks [44] such that the nodes should cooperate to establish a network, and gather and transfer the data, calling for efficient topology management solutions. Clustering is recognized as a popular technique for this purpose, e.g., to improve efficiency of routing methods and reduce energy consumption due to wireless data transmission using hierarchical data fusion [17]. From about 2000 to 2019, tens of WSN clustering techniques have been proposed. In this section, we study all significant clustering methods by reviewing around 125 papers. Unlike other survey papers, we do not focus on the design details of clustering methods like algorithm complexity, methodology, etc. as it is not the goal of our study. We review the most significant clustering techniques in WSNs based on their objectives and the specific properties of networks. Considering the former, the primary goal of clustering in WSNs is retaining and/or improving a pre-defined set of quality-driven objectives. A clustering technique is designed to support one or multiple objectives simultaneously. The objectives include:

- Energy Consumption (E)

- Fault Tolerance (F)

- Physical Layer Support (U)

- Throughput (T)

- Coverage (O)

- Mobility Management (M)

- Packet Delivery Ratio (Y)

- Mutli-Sink Support (K)

- Number of Packets Received by BS (P)

- Load Balancing (L)

- Reliability (R)

- Jitter (J)

- Scalability (A)

- Connectivity (C)

- Stability (B)

- Security (S)

- Delay (D)

We also consider a set of network and clustering properties in our review that are crucial for the applicability of clustering techniques to IoT networks. These properties include: heterogeneity, role of $\mathrm{CH}$, inter-cluster routing, and mobility. Below, we explain the reasons for choosing these properties.

Heterogeneity: Contrary to WSNs which are often homogeneous [19], IoT networks are known to be heterogeneous [3]. Thus, network management in IoT needs to deal with nodes heterogeneity as it affects the performance of the network from different aspects, e.g., stability, connectivity, and QoS [45], [46]. Clustering is a network management technique that can address heterogeneity issues in the network. Thus, WSN clustering techniques that support heterogeneity are more compatible with the nature of IoT networks.

Role of CH: Generally, CHs in WSNs are not in charge of performing complicated tasks, thus the parameters to select $\mathrm{CHs}$ are often limited to energy and data forwarding resources. However, some clustering techniques utilize $\mathrm{CHs}$ for data fusion, in which the $\mathrm{CH}$ selection criterion is the computation power of nodes [15]. Such techniques can be suitable for clustering in IoT networks as $\mathrm{CHs}$ in IoT can be used to execute computationally demanding tasks [47], [48], e.g., edge-level image processing in smart cities.

Inter-cluster routing: In some WSN clustering techniques, data transmission to the sink is performed through a 2-hop communication model (i.e., node to $\mathrm{CH}$ and $\mathrm{CH}$ to sink) over a multi-point to point network infrastructure. However, many other clustering techniques allow data routing with more hops between the sources and the sink. To apply existing WSN clustering techniques to IoT, such techniques would be more advantageous because in IoT networks, services can run inside the network on intermediary nodes, e.g., hierarchical data processing in Fog [49]. In addition, the number of gateways may be more than one node in IoT, implying the need for intercluster routing to access the most efficient gateway in terms of, e.g., bandwidth and delay. It should be noted that we do not consider intra-cluster routing as a parameter in applicability of WSN clustering techniques to IoT as it is used in special cases such as in blind spaces or for big-sized clusters, in addition to the fact that it may also degrade QoS.

Mobility: Based on their applications, IoT networks may contain more mobile nodes [50] than WSNs [51], e.g., wearable devices and IoV. Clustering techniques that support mobility would be more appropriate for IoT, thus we include mobility as a network property in reviewing WSN clustering techniques.

Table I lists the studied WSN clustering techniques, sorted in chronological order. Each technique has been examined based on the aforementioned network properties and objectives. For example, LEACH [12], proposed in the year 2000, neither supports heterogeneity, nor mobility. CHs in this method are mainly in charge of data fusion and they use direct inter-cluster routing to transmit data to BS. In addition, the sole clustering objective of LEACH is to reduce energy consumption (E). Some techniques support objectives that are not among the main 17 objectives. For them, the table includes a short description of the targeted objectives, e.g., EACLE [52] reduces packet collision, in addition to reducing energy consumption (E). Moreover, for techniques supporting heterogeneity and mobility, the table clarifies how these properties are supported. If there is no comment for a technique supporting heterogeneity, its heterogeneity aspect is by default the energy resource. The last row of Table I shows the statistics of the studied techniques. As shown, most of the literature focuses on improving the energy consumption, in addition to load balancing and scalability. Moreover, it indicates that many techniques support multi-hop inter-cluster routing. With respect to the network properties, there are not many clustering techniques supporting heterogeneity and mobility. As these are two most important parameters in IoT clustering, more research effort in this area is needed.

To the best of our knowledge, this table compiles the most well-known clustering techniques in WSNs, their properties and objectives. It allows filtering the techniques based on the desired properties and objectives for clustering. Regarding their applicability to IoT networks, it allows finding the suitable techniques based on the chosen network properties and clustering objectives. In the next section, we study those types of techniques, in the context of IoT, according to the aforementioned clustering objectives, and discuss existing IoT clustering solutions addressing each objective.

In Table II, objectives of clustering are reviewed based on their correlation. The white cells on the diameter of the table show the number of techniques that support an objective on $\mathrm{x} / \mathrm{y}$-axis, e.g., 44 techniques support load balancing (L). Other numbers, in each column, show the percentage of the techniques that support an objective on the y-axis. 


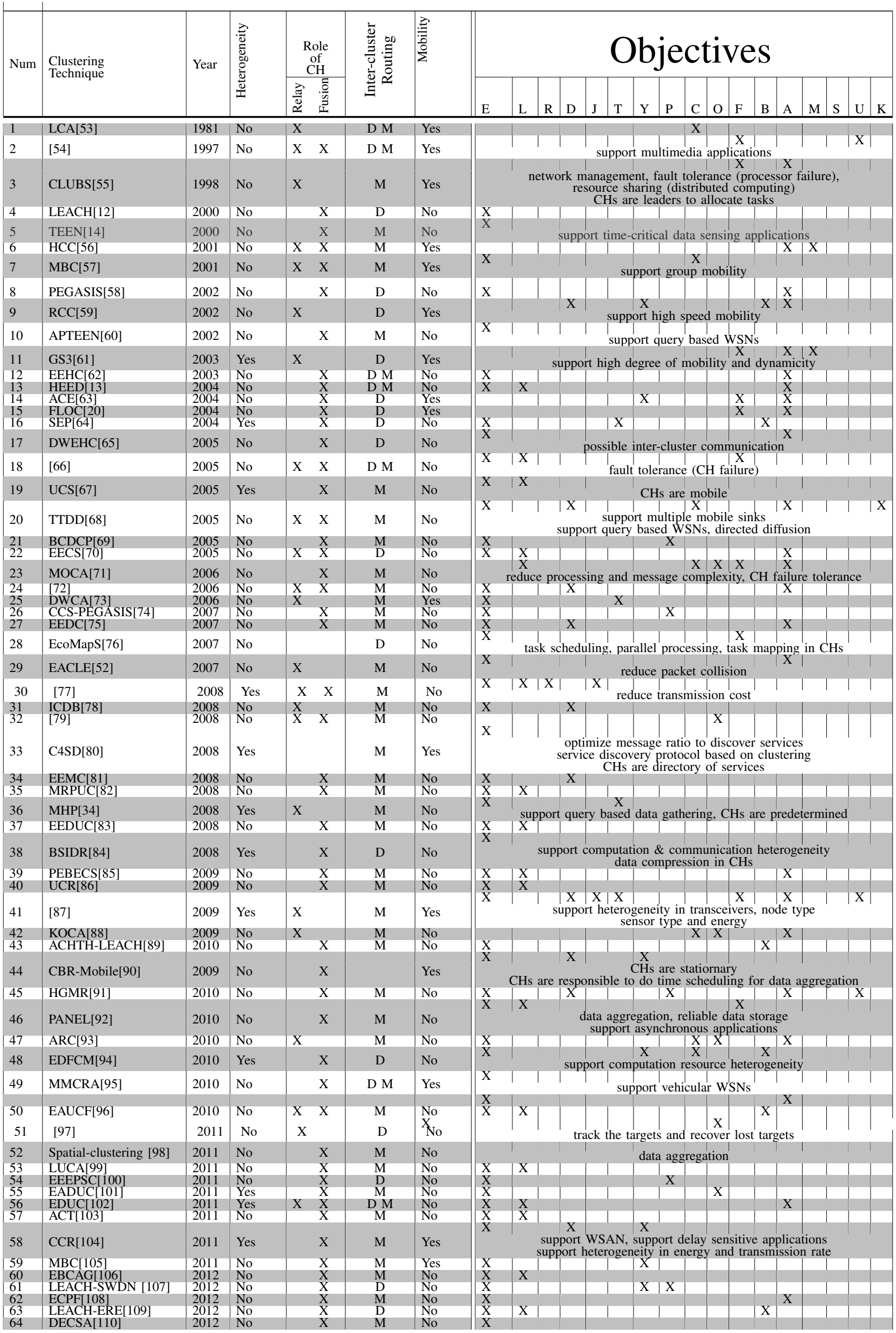




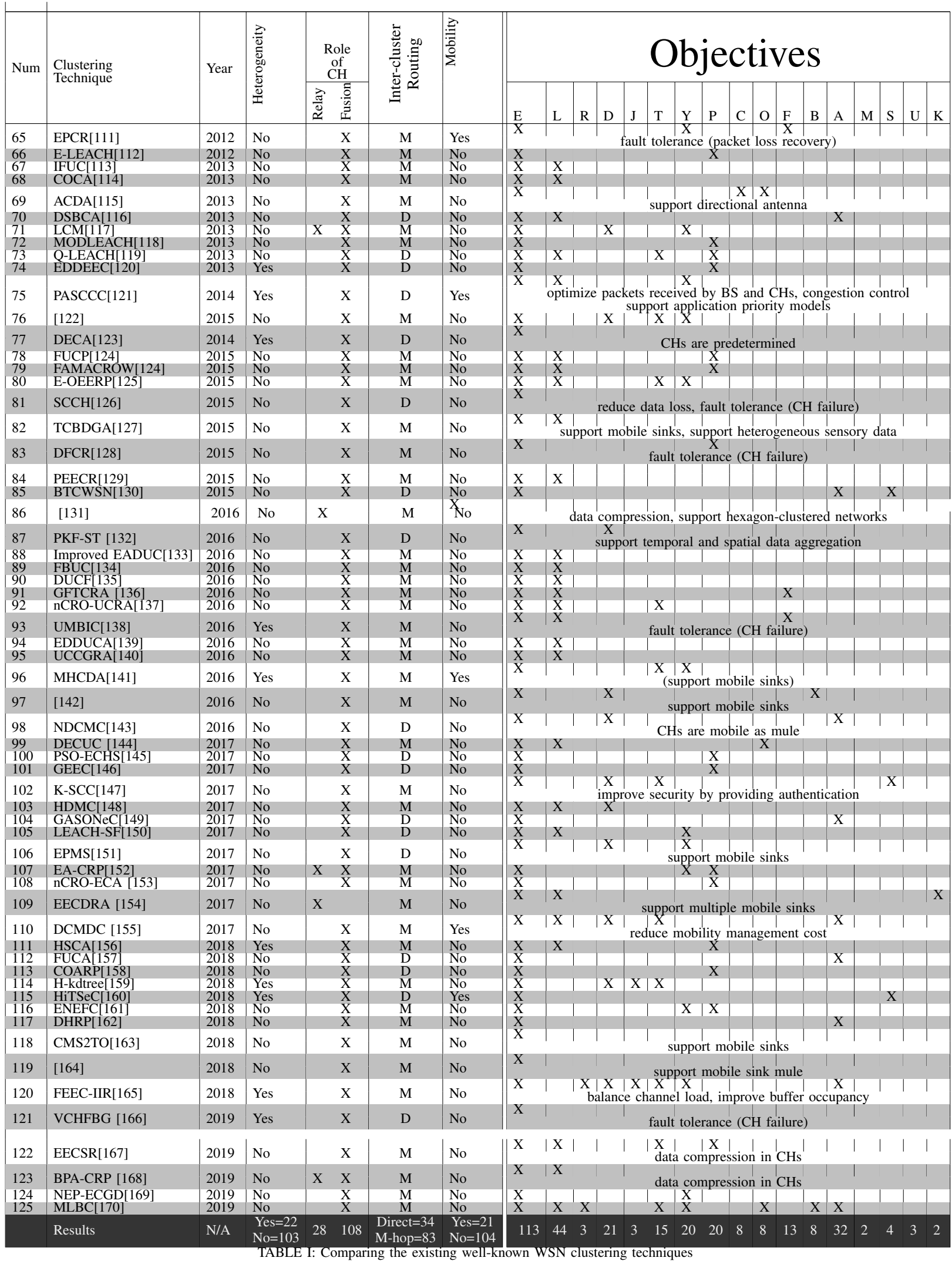


For example, out of 44 papers on $L, 97,7 \%$ of them support $E$ as other objective, while only $4,5 \%$ of them support $R$. The heatmap highlights which pairs of objectives in existing clustering techniques have received more attention by the researcher. In addition, the statistical analysis of Table I shows that the number of papers that support $1,2,3,4,5,6,7$ and 8 objectives simultaneously are $15,53,40,10,4,0,2$, and 1 , respectively. This indicates that clustering techniques are normally used to support multiple objectives simultaneously, where the majority of them support two objectives.

The other useful insight is to find out which pairs of (objective, networkproperty) have been considered more in the design of clustering techniques. For this purpose, out of the 125 techniques in Table I, we counted the number of techniques that support a given (objective, networkproperty) as shown in Table III, e.g., 92 of the techniques with energy efficiency (E) as an objective can not support heterogeneity. As shown in the table, most of the literature does not support heterogeneity. Considering the role of $\mathrm{CH}$, the table shows that data fusion has been the common role of $\mathrm{CH}$ in achieving most of the objectives such as $E, L, Y$ and $P$. Besides the obvious reason of fusing data for energy consumption reduction or load balancing, for $Y$ and $P$ sending fused data instead of individual data items will lead to a lower rate of data loss in the network. Considering routing, multi-hop methods are quite common for achieving $E$, in addition to improving network coverage (O) and connectivity (C). In addition, it can be seen that there is a limited amount of literature that supports mobility when the objective is energy consumption improvement or load balancing (13 and 2 respectively). This implys that mobility support can disrupt achieving these popular objectives. The above statistical evaluation shows that designing clustering techniques for IoT networks needs more attention as supporting mobility and heterogeneity are two key requirements for IoT clustering.

\section{Migrating FROM WSNS TO IOT}

WSNs essentially connect sensor nodes to the Internet through a gateway, contrary to smart devices in IoT that are often able to connect directly to the Internet. Figure 2 shows different types of IoT networking models. In centralized networking solutions, nodes connect to on-premise servers or cloud platforms to process data. By integration IoT with cloud computing platforms, smart devices connect to virtually infinite resources to store and process data centrally. In the decentralized model, Machine-to-Machine (M2M), D2D, and ad-hoc networks allow IoT networks to connect nodes directly. The main difference between D2D and M2M is the physical proximity of devices. In D2D a pair of devices must be in close physical proximity, but in M2M they can be distant, but they are able to communicate directly without the need for a central node (e.g., servers). Communication technologies such as wide area networks and cellular networks have also further realized the decentralized networking model in IoT, such as LoRA [171] and NB-IoT [171], respectively. To conclude, many IoT networks resemble ad-hoc networks with respect to topology and the communication model. Moreover, IoT is rapidly being introduced for massive scale applications such as smart cities and vehicular networks, which are often built over ad-hoc infrastructures, as mentioned above.

Contrary to the centralized models with the capability of direct connectivity to the server or the Cloud, in the decentralized model, topology management is a critical requirement for efficient and scalable management of IoT networks. Being the primary solution for topology management, clustering can be applied to decentralized IoT networks in order to improve resource usage, QoS, and load balancing, to name a few. Therefore, the survey is carried out for this type of IoT networks.

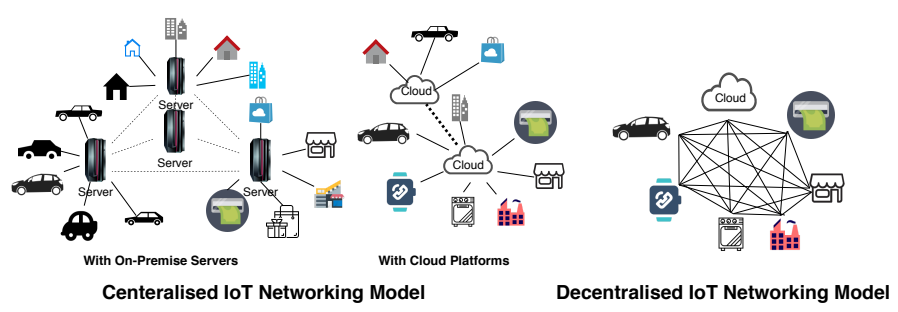

Fig. 2. General IoT networking models

\section{A. Clustering in WSNs vs. in IoT: Technical Differences}

Existing solutions and technologies for WSN clustering have the potential to be applied to IoT networks. However, clustering in IoT introduces new challenges that are discussed below. The summary of the technical differences is presented in Table IV.

1) Heterogeneity: A fundamental challenge in IoT is supporting heterogeneity. Compared to WSNs, IoT network management techniques need to consider heterogeneity as a contingent characteristic of the network. In IoT, in addition to WSN nodes, other types of smart devices (e.g., smartphones and cameras) may be used in one application scenario [172]. Even compared to typical WSNs, WSNs-assisted IoT [173] are essentially different in terms of heterogeneity [174]. As shown in Table I, most WSN clustering techniques are based on homogeneous networks. Those supporting WSN heterogeneity focus mostly on energy resources [175], but in IoT, heterogeneity is somewhat different with respect to clustering:

- Energy: Heterogeneous WSN clustering techniques (HeWCTs) mostly support heterogeneous networks that have predetermined initial energy, though all nodes have not the same initial energy. However, they have not considered the networks with high diversity in node energy levels, or nodes with rechargeable energy resources. The fact is that, In IoT networks, nodes may have different energy capacities, ranging from nodes with unlimited energy resources, e.g., plugged PC or cameras, to nodes with flexible energy resources, e.g., outdoor sensors equipped with photovoltaic solar panels.

- Computing power: Similar to heterogeneity in energy, in some cases, clustering techniques in HeWCTs support a few predefined levels of computing power like in [94]. However, in heterogeneous IoT (HetIoT) [3], the smart devices may have divers computing power levels. Such diversity can 
TABLE II

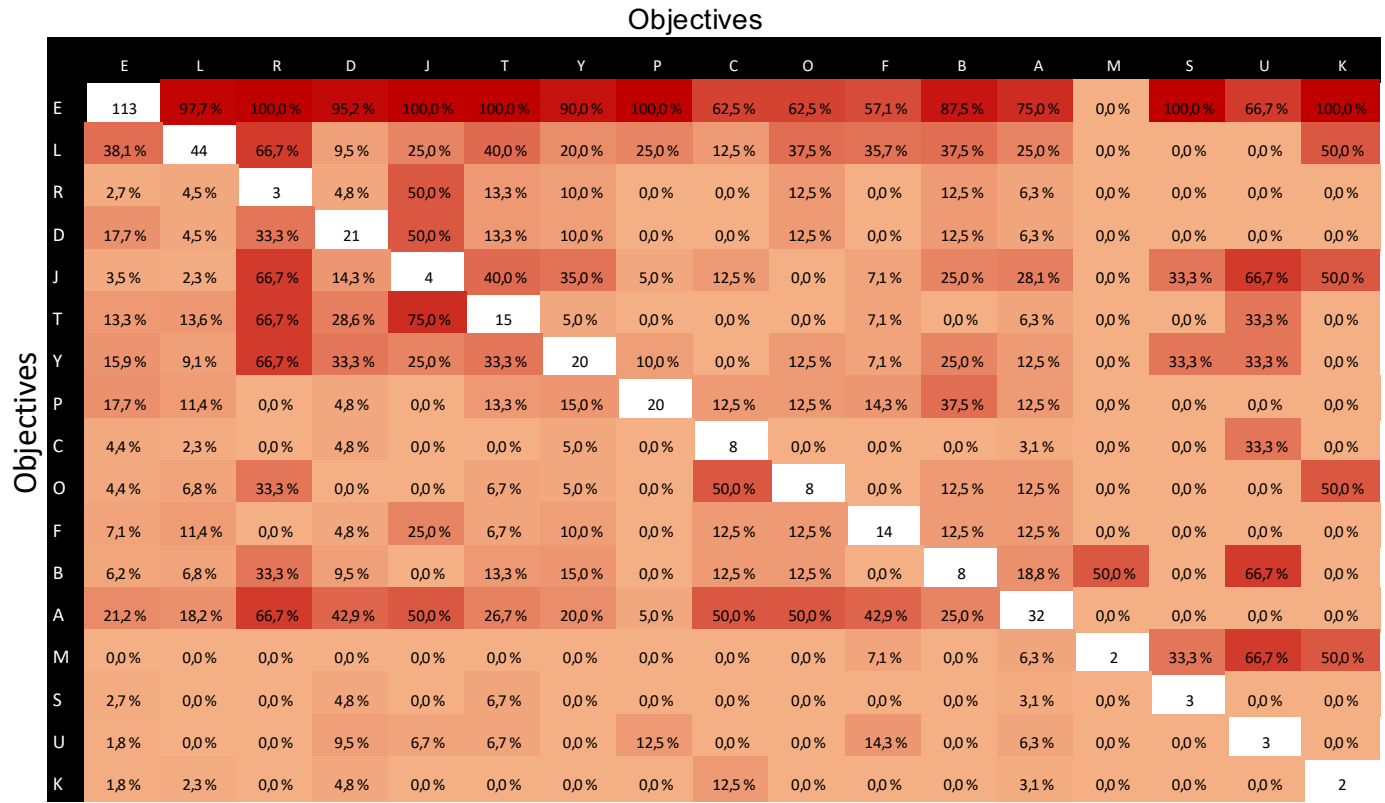

TABLE III

THE CORRELATIONS BETWEEN OBJECTIVES AND PROPERTIES OF THE NETWORK IN THE REVIEWED CLUSTERING TECHNIQUES

\begin{tabular}{|c|c|c|c|c|c|c|c|c|c|c|}
\hline & \multicolumn{2}{|c|}{$\begin{array}{c}\text { Support } \\
\text { Heterogeneity }\end{array}$} & \multicolumn{3}{|c|}{ Role of CH } & \multicolumn{3}{c|}{ Routing } & \multicolumn{2}{|c|}{ Mobility } \\
\hline & Yes & No & Fusion & Relay & Both & Direct & Multi-hop & Both & Yes & No \\
\hline E (113) & 21 & 92 & 101 & 20 & 11 & 30 & 77 & 5 & 13 & 100 \\
\hline L (44) & 6 & 38 & 43 & 7 & 6 & 6 & 34 & 3 & 2 & 42 \\
\hline R (3) & 2 & 1 & 3 & 0 & 0 & 0 & 3 & 0 & 3 & 0 \\
\hline D (21) & 4 & 17 & 17 & 6 & 3 & 4 & 17 & 0 & 5 & 16 \\
\hline J (4) & 4 & 0 & 3 & 2 & 1 & 0 & 4 & 0 & 1 & 3 \\
\hline T (15) & 6 & 9 & 12 & 3 & 0 & 2 & 12 & 0 & 4 & 11 \\
\hline Y (20) & 5 & 15 & 18 & 2 & 4 & 7 & 13 & 0 & 8 & 12 \\
\hline P (20) & 2 & 18 & 20 & 1 & 1 & 7 & 12 & 0 & 0 & 20 \\
\hline C (8) & 1 & 7 & 5 & 5 & 2 & 1 & 6 & 1 & 2 & 6 \\
\hline O (8) & 1 & 7 & 6 & 3 & 1 & 0 & 8 & 0 & 0 & 8 \\
\hline F (14) & 3 & 11 & 9 & 6 & 2 & 5 & 7 & 2 & 7 & 7 \\
\hline B (8) & 2 & 6 & 7 & 2 & 1 & 4 & 4 & 0 & 1 & 7 \\
\hline A (32) & 4 & 28 & 25 & 12 & 5 & 12 & 17 & 3 & 8 & 24 \\
\hline M (2) & 1 & 1 & 1 & 2 & 1 & 1 & 1 & 0 & 2 & 0 \\
\hline S (3) & 1 & 2 & 3 & 0 & 0 & 2 & 1 & 0 & 1 & 2 \\
\hline U (3) & 1 & 2 & 2 & 2 & 1 & 0 & 2 & 1 & 2 & 1 \\
\hline K (2) & 0 & 2 & 1 & 2 & 1 & 0 & 2 & 0 & 0 & 2 \\
\hline
\end{tabular}

make clustering more complicated when combined with other clustering criteria for, e.g., selecting $\mathrm{CH}$ and assigning tasks. Nodes with high computing power are a potential candidate to gather, fuse and process data.

- Storage: Having smart devices with different amounts of data storage in the network is another aspect of heterogeneity that should be taken into account in IoT network clustering, in particular because of the big data applications of IoT [176]. To the best of our knowledge, there is only one clustering technique [177] that supports heterogeneity in storage.

- Network interface controller (NIC): In IoT networks, nodes may be equipped with different network interfaces such as Wi-Fi, Zigbee, XBee, Bluetooth, BLE, and LTE. In HeWCTs, researchers consider mainly low-power communication technologies such as Zigbee and 802.15.4 [111]. Considering clustering, network nodes with different types of interfaces can pose two challenges: $i)$ they may not be discoverable by each other in physical proximity [87], and ii) in inter- and intra-cluster routing there is a trade-off in choosing the best network interface with respect to, e.g., cost, energy, transmission range, etc. [87].

2) Mobility: Supporting mobility of nodes in IoT networks has been an important issue, e.g., in MANET-IoT networks [178]. The authors in [179] review mobility management challenges in IoT and show how mobility can make IoT networks unstable. Mobility in WSNs has often been proposed for rather simple scenarios such as mobile sinks, unlike highly dynamic IoT applications such as smart cities [5], and V2V [180]. Additionally, mobile nodes not only change their locations in the IoT network, but also may switch between different networks which is related to handover mechanisms in IoT networks [181][182]. These issues make designing clustering techniques in IoT networks challenging [183].

3) Device Identity: The other issue is that the IoT network includes not only ID-based devices but also IP-enabled devices [184]. This implies that clustering techniques should be designed in such a way that they can support clustering both these types of nodes. For instance, IP-enabled devices can basically communicate with ID-based devices, while the opposite direction of communication may not be straightforward [185]. Therefore, clustering techniques can adopt two different approaches for clustering: clustering devices with the same network type, or clustering both IP-enabled and IDenabled devices.

4) Device-to-Device (D2D) Communication: D2D communication involves direct short-range communication between IoT devices without the support of network infrastructures, such as BSs or access points [186]. Many IoT applications and services are realized through D2D communication networks such as Zigbee, Bluetooth Low Energy (BLE), Radio Frequency Identification (RFID) and Near Field Communication 
TABLE IV

COMPARING THE TECHNICAL DIFFERENCES OF WSNS AND IOT TO USE CLUSTERING

\begin{tabular}{|c|c|c|c|}
\hline \multicolumn{2}{|c|}{ Technical Differences } & WSNs & IoT \\
\hline \multirow{4}{*}{ Heterogeneity } & Energy & Occasionally & Common \\
\hline & Computing Power & Selodm & Common \\
\hline & Storage & Seldom & Common \\
\hline & NIC & Mostly single NIC & Common Multi-NIC \\
\hline \multicolumn{2}{|c|}{ Mobility } & Occasionally & Very common \\
\hline \multicolumn{2}{|c|}{ Communication Type } & Mostly ad-hoc & Possibly ad-hoc \\
\hline \multirow{2}{*}{ Applications } & Deployment model & Single application & Multi-application \\
\hline & Design model & data-centric & Service-based \\
\hline
\end{tabular}

(NFC). 6LoWPAN is an advanced D2D enabling technology (the scaled-down version of the IPv6 standard for LoWPANs) [187]. Given the wide usage scope of 6LoWPAN, IoT clustering can take advantage of D2D communication through 6LoWPAN and exploit the flexibility and diversity of D2D communications to provide more efficient and applicationdriven clustering techniques.

5) Applications: Although clustering is targeted toward the network layer requirements, there are challenges, raised by IoT applications, that should be considered in IoT clustering:

- Deployment model: Contrary to WSNs which usually host a single application [188], IoT network infrastructures may be offered to host multiple applications with their own quality requirements. As most WSN-based clustering techniques rely on the network layer parameters for clustering, e.g., energy, supporting application-level requirements by clustering is crucial, in particular when applications' requirements are in conflict with each other, e.g., QoS and resource demands.

- Design models: Unlike WSNs which are mostly data-centric, IoT applications are often service-based [1], in which services can run on various devices and platforms, from local computing platforms to the Cloud. To further facilitate IoT services development and improve their efficiency, new computing technologies such as Edge/Fog computing [189], [2] have been recently emerged. With respect to the network infrastructure, Fog/Edge can possibly change the essence of the network topology from multi-point to point (like in WSNs) to multi-point to multi-point due to distribution of IoT services across the network. This will pose new challenges in designing IoT clustering routing algorithms and $\mathrm{CH}$ selection as they have to adhere to the target service distribution model.

\section{From WSNs to IoT: CONVEntional Clustering OBJECTIVES}

A clustering technique can support a single objective, or multiple objectives simultaneously. The IoT networks that resemble ad-hoc networks share several commonalities with WSNs regarding conventional quality-driven objectives. The nature of ad-hoc IoT introduces new challenges in achieving clustering objectives in IoT networks. In this section, we study each objective in detail. In particular, for each objective and the associated general clustering solutions, we discuss existing IoT clustering techniques, and also design concerns raising due to applying clustering to IoT for that specific objective. It should be noted that, due to the high volume of the literature in
Fig. 3. All conventional clustering objectives and exiting solutions

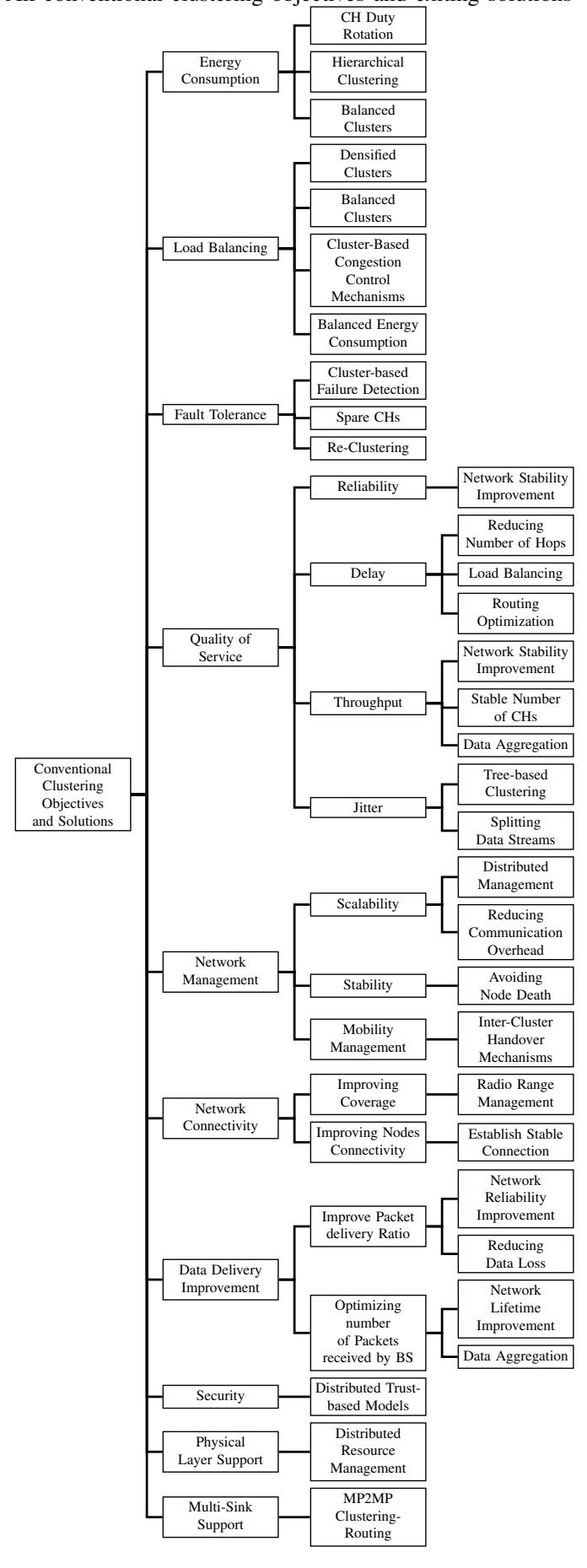

clustering, we split existing solutions to pure WSN clustering techniques, and IoT-related clustering techniques (i.e., WSNassisted IoT and pure IoT). The former category is compiled as a list in Section III, while the prominent works in the latter category are discussed per objective in this section.

Before discussing the objectives in detail, in Figure 3 we illustrate an overview of of clustering objectives and existing solutions. Figure 4 also shows the challenges in achieving those objectives in IoT through clustering techniques. These figures together provide a brief overview of what we present in this section. 
Fig. 4. IoT-Specific concerns in achieving clustering objectives

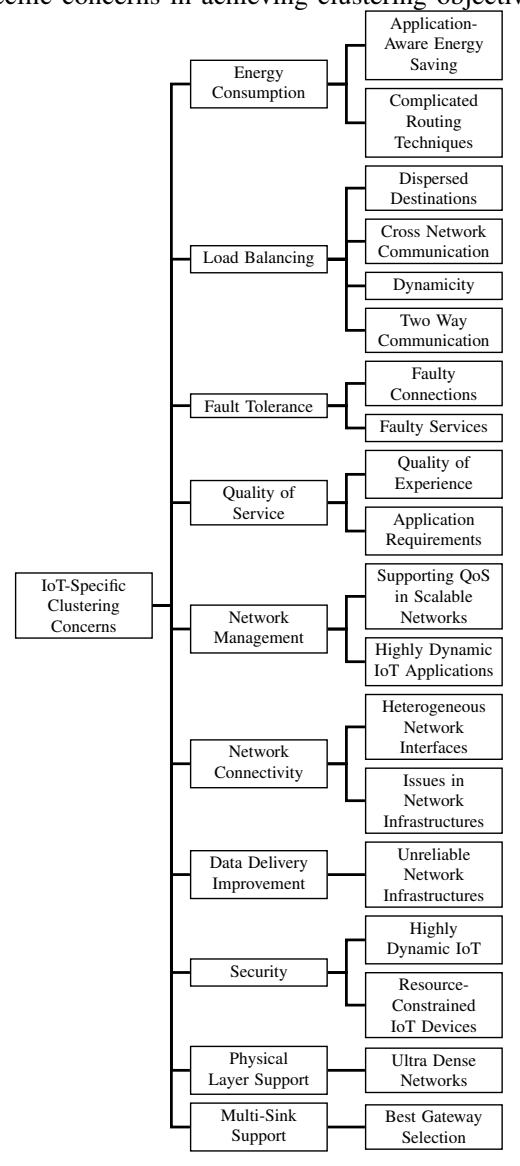

\section{A. Energy Consumption Improvement}

As the most important objective of clustering, clustering techniques can balance energy consumption and improve the network lifetime. Since data communication is the prominent source of energy consumption in low-power networks [37], clustering can mitigate it through inter/intra-cluster routing by middle nodes, as well as data aggregation in CHs.

\section{Existing solutions and techniques include:}

- $\mathrm{CH}$ duty rotation: In clustering techniques, $\mathrm{CH}$ s consume a large amount of energy compared to cluster members [190]. As a multi-objective selection technique, $\mathrm{CH}$ selection is a complicated task which can be performed using Fuzzy logic [191] [192], AI methods [193], and heuristic methods [194] [195]. CHs as a central node should support various objectives of networks such as QoS, reliability, and balancing energy consumption. $\mathrm{CH}$ rotation-rotating $\mathrm{CH}$ duty-is a common solution to achieve such objectives. One key criterion for triggering $\mathrm{CH}$ rotation is the threshold for energy consumption or the remaining energy of nodes. In homogeneous networks, $\mathrm{CH}$ duty rotating is a more complicated task because all nodes have the same energy resources [36]. On the contrary, in heterogeneous networks, $\mathrm{CH}$ re-selection would be easier thanks to the presence of energy-rich nodes limiting the number of $\mathrm{CH}$ node candidates. Being highly heterogeneous compared to WSNs [3], $\mathrm{CH}$ rotation is more natural in IoT, keeping the performance of the network at the expected level. In Heterogeneous IoT
(HetIoT), more replacement candidates are available when looking for a node for data forwarding (aggregated) or processing energy-intensive tasks. In [196], the authors use the k-means algorithm in IoT networks based on the residual energy to select new CHs. EnergIoT [197] is a hierarchical clustering method to assign duty cycle (i.e., switching between sleeping and active modes) ratios to devices for improving energy consumption. However, in IoT, CHs are not only in charge of data aggregation and forwarding but also responsible to perform more complicated tasks such as hosting services, data compression, network management, etc. This makes $\mathrm{CH}$ selection more complicated as the residual energy cannot be the only parameter for selecting the new $\mathrm{CH}$ like in WSNs. Therefore, $\mathrm{CH}$ selection becomes a multi-objective problem with new parameters such as location and density of the nodes in Ultra Dense Networks (UDN) and computation power, network interfaces, and storage in HetIoT. In [198], the authors use the residual energy of IoT nodes together with the density and location of alive nodes as the parameters to rotate duty of $\mathrm{CH}$.

- Hierarchical clustering: This is a method that organizes the network in a number of layers. Each layer can communicate with neighbor layers, which saves more energy compared to transferring data directly to far destinations. In hierarchical clustering techniques, middle nodes in upper layers (closer to the BS) consume more energy because of forwarding massive volume of data from the lower layers to the upper layers. One popular method to balance the load is establishing hierarchical unbalanced clusters. Clusters in the lower layers have more members compared to the upper layers [152]. Since receiving data consumes a fixed amount of energy, $\mathrm{CHs}$ of lower layers can have more members but do not need to forward/aggregate many packets from the other layers; therefore unbalanced layers/clusters would be an appropriate option to balance energy consumption. In IoT networks, hierarchical clustering has been exploited to balance energy consumption. In [199], Tang et al. introduce a hierarchical clustering index tree based on EnerGy-eFficiency Hierarchical Clustering index tree (ECH-tree) that organizes regions, called grid cells in IoT networks. The method ensures that the upper level grid cells have less dead spaces as compared to the lower levels. Using this technique, the energy consumed to collect and forward the data is reduced as compared with traditional index tree based techniques. In [200], the authors introduce hierarchical unbalanced layers for industrial IoT networks to balance the energy consumption as the layers closer to BS contain smaller clusters.

- Balanced clusters: Although unbalanced clustering (through hierarchical clustering) is a solution for balancing energy consumption, it would be challenging in ad-hoc networks in which nodes are mobile or not uniformly distributed. Unbalanced clusters in such networks can lead to more energy consumption due to gathering data from many member nodes. In such cases, to have fair energy consumption, clusters should be balanced based on the number of nodes and the volume of generated data, not the size of the areas covered by the clusters [201]. In IoT, balanced clusters have 
been proposed to improve energy and resource consumption. In [200], Zhang et al. introduce a method that partitions industrial IoT networks into grid clusters in each layer to balance energy consumption. The size of the grid clusters are calculated by solving polynomials. In [202], the authors introduce a service-aware clustering method to improve the energy consumption. The proposed method uses a traffic engineering model to design the clustering technique and provide profiles of services running on sensor nodes to improve the network lifetime.

IoT-specific concerns. Whereas the above techniques have been somewhat considered for energy saving in IoT, there are a number of IoT-specific issues that should be studied when applying clustering for energy saving in IoT, as discussed below:

- Application-aware energy saving: From a different viewpoint, unlike WSNs, IoT applications and their services can affect the design of clustering techniques and energy consumption improvement as the nodes may connect to different hosts (local or remote) for service invocation. While in many WSN network management techniques, data-aware energy consumption has been neglected [148], IoT services serve as a new factor in energy consumption measurement [203], thus designing a clustering technique cannot be performed only by considering the network layer requirements [204] [205]. In [206], the authors introduce a clustering method for multimedia IoT, based on the $\mathrm{K}$ means algorithm. They establish a tree to divide multimedia sessions and connect clusters of nodes to support the requirements of multimedia applications.

- Routing techniques: The presence of different network interfaces in IoT makes the implementation of clusteringbased routing techniques complicated-as a solution to reduce energy consumption. Specifically, each node can have multiple network interfaces with different energy consumption in data transmission, connection speed, and cost. In addition, as IoT networks generally contain more gateways due to their scale, data volume, and QoS requirements [5], routing protocols should support point-to-point, point-tomultipoint, multipoint-to-point and multipoint-to-multipoint communications, making clustering-routing techniques more complicated compared to WSNs. Routing Protocol for LowPower and Lossy Networks (RPL) as the standard routing protocol has been introduced to solve the problem of IoT routing [207], [208], but there are doubts that RPL is able to satisfy requirements of IoT in real world applications [209].

\section{B. Load Balancing}

Load balancing is the second most important conventional objective of clustering. Generally, clustering techniques use a type of divide and conquer method to transfer data from nodes to BSs. A high load of data to transfer can result in unbalanced resource consumption and influence QoS support. Load balancing can also solve the "Hot Spot problem" which is a common problem in WSNs [210] [164] and IoT [211]. The Hot Spot problem is a situation in which some nodes in the network transfer high volume of data compared to others which cause network congestion. Generally, load balancing is a critical issue in IoT due to the massive scale, huge volume of data and mobility of IoT networks which can cause inefficiency, e.g., in [212] a technique is introduced to balance the load of the network to reduce interference among nodes ${ }^{1}$. Existing solutions and techniques include:

- Densified clusters: means creating more clustering layers and establishing more clusters in each layer. Having more $\mathrm{CHs}$ will allow to distribute the network load among more destinations (middle nodes). Moreover, having more layers in hierarchical clustering can extend the number of hops as in each layer more nodes are available as the next hop for data transmission [214]. Considering layering in IoT, the authors in [215] propose a multi-path routing based on clustering techniques, providing more routes for areas that need to transfer higher volume of data.

- Balanced clusters: Balancing clusters in terms of volume of generated data would be a solution especially in direct communication. If nodes are homogeneous, the number of nodes can be a parameter to balance the load among clusters [216]. As an example, in [217] the authors introduce a Software Defined Network (SDN) model to establish a Particle Swarm Optimization (PSO) based clustering table that is used by the SDN controller to balance the clusters, resulting in load balancing. The authors in [218] integrate a clustering technique with compressive sensing in IoT. They introduce the CSLB-CS method which is a new cluster sizebased load balancing to optimize compressive sensing in IoT-based sensor networks. In heterogeneous networks like IoT, the packet rate per node can be a parameter to balance clusters, but the packet rate is a challenging and resourceconsuming parameter to predict and process on-the-fly as it needs network traffic classification algorithms [219].

- Congestion control mechanisms: Some clustering techniques are aimed to address the congestion control problem in the network, resulting in load balancing by dividing the network load among existing resources. In [220], the authors introduce a clustering technique to solve the congestion control problem in M2M IoT networks, resulting in load balancing. They assume the IoT network is based on M2M communication in which clustering provides a slot allocation mechanism based on Q-Learning to avoid congestion. In [221], the authors propose a clustering technique to manage multimedia big data in mobile IoT. They propose a new approach in MANET-IoT based on a modified LEACH clustering method. They also propose a congestion control method by link utilization based on the link status.

- Balanced energy consumption: As data transmission is the most important cause of energy depletion, fair distribution of energy consumption among nodes can indirectly improve load balancing and vice versa. In [222], the authors introduce a clustering technique that balances the network load based on a cost function. LiMCA is a IoT clustering technique, introduced in [223], to balance the energy consumption in $\mathrm{CHs}$ and members by training them about their

\footnotetext{
${ }^{1}$ In [213], a comprehensive review of load balancing techniques in IoT including clustering-based techniques, is presented.
} 
coarse-grain location. They analyze energy consumption by evaluating the lognormal shadowing channel, inter-cluster and intra-cluster traffic, and the packet error rate. They consider that both $\mathrm{CH}$ and members of clusters have a predetermined location based on the stochastic deployment scheme.

IoT-specific concerns. In order to use clustering for load balancing in IoT, we face a number of challenges:

- Dispersed destinations: Concurrent applications need to support different rates of packet streams with various volume of data. Moreover, by using new emerging technologies such as Fog and Edge, destinations of streams cannot be only BS(s) or gateways, but also other nodes in the network [224]. This transforms the communication model in IoT networks to a multipoint-to-multipoint model, making load balancing challenging [225].

- Cross network communication: Considering load balancing, the network beyond the gateway can not be controlled. For example, to support low latency in communication, packet streams may be re-routed to a more efficient gateway, leading to dynamic unbalancing of the network load. In [226], the authors introduce a new idea called Cognitive IoT gateways, which can decide which services should be migrated from the Cloud to the IoT network and vice versa, dynamically. Using Cognitive gateways, nodes can monitor the network performance such as QoS and load balancing and bring services from outside of the network to inside.

- Dynamicity: In IoT, consumers and providers of services may not be known in advance, appearing and disappearing over application lifetime, e.g., multimedia applications. Unforeseeable service requests can make the IoT nodes more unpredictable with respect to the data load [227]. As the load of the network is dynamic, load balancing should be monitored on a regular basis and optimized accordingly.

- Two-way communication: The most crucial difference between WSNs and IoT which affects the network load balancing is related to one-way and two-way communications. To balance the network load, two-way communication should be considered in IoT and hops should allocate resources to forward data in both ways.

\section{Fault Tolerance}

There are different causes for node failure in IoT and WSN networks, such as battery depletion, or failure in hardware components like transceiver and processor which can be damaged by external factors. Moreover, connectivity failure can happen by physical or environmental factors, mobile nodes, or faulty nodes. To handle such types of failures, fault-tolerant clustering techniques have been introduced to replace faulty nodes with other nodes and keep the network stable [166].

Existing solutions and techniques include:

- Cluster-based failure detection: The speed of failed node detection methods is a challenge because high latency in detecting failures can cause data loss especially in dataloss sensitive applications. In addition, connections should be checked to determine any fault in connectivity which can cause delay or data loss. In both cases, clustering can help to detect failures and recover the infrastructure quickly. In [228], the authors introduce a routing method for IoT networks based on the bio-inspired particle multi-swarm optimization (PMSO) strategy which is able to detect faulty nodes quickly and replace routes with new ones. They use super nodes serving as a $\mathrm{CH}$ for information gathering.

- Spare nodes (CHs): To tolerate faulty nodes, some techniques use spare nodes to take over responsibilities and avoid data loss. Spare $\mathrm{CHs}$ can be replaced by faulty $\mathrm{CHs}$ as Hot Spots and solve the problem efficiently. In [166], a method is introduced to select failure-free $\mathrm{CHs}$ to replace faulty $\mathrm{CHs}$ in IoT. It forms a virtual $\mathrm{CH}$ that includes three failure-free $\mathrm{CHs}$ based on the Flow-Bipartite Graph Modeling and energy of all failure-free $\mathrm{CHs}$. After detecting a $\mathrm{CH}$ failure by $\mathrm{BS}$, BS will ask failure-free $\mathrm{CHs}$ to take over the responsibilities of the faulty $\mathrm{CH}$.

- Re-clustering: As spare nodes induce overhead in selecting and keeping them updated, the simplest solution is reclustering. After detecting failures, clusters can be destroyed and re-established, recovering the network infrastructure to transmit data. In [229], the authors introduce a clustering based routing technique for IoT networks which replaces faulty $\mathrm{CHs}$ with vice $\mathrm{CHs}$. In case of faulty vice $\mathrm{CH}$, reclustering will be performed.

IoT-specific concerns. In IoT, fault tolerance is a big challenge because of being highly dynamic and heterogeneous and also unreliability of the IoT network infrastructure [230] [231] [232]. Addressing IoT fault tolerance issues by clustering introduces the following challenges:

- Faulty connections: In IoT, faulty connections are common [233]. Reliable communication mediums, such as WiFi and $4 \mathrm{G}$, can reduce faulty connections, but technologies such as cellular-D2D and BLE still suffer from communication problems [6]. Moreover, mobile IoT devices can increase the probability of connection faults in IoT networks. These issues can make the clusters and routes between nodes unstable resulting in, e.g., data loss.

- Faulty services: Services are the other source of faults in IoT [234], [235]. They can disrupt the network function, in particular in the case of clustered networks. For example, one solution for handling faulty services is to run the services in other nodes [236], but it can influence the efficiency of the clustered network as the routes should be re-established, affecting load balancing [237].

\section{Quality of Service}

QoS is a rather a broad topic, encompassing divers quality aspects of network services, such as packet loss, bit rate, throughput, transmission delay, availability, jitter, etc. Although WSNs and IoT share many QoS concerns, the specific characteristics of IoT networks introduce new QoS requirements, such as guaranteeing QoS in simultaneously running IoT applications, and higher delay-sensitivity in safety-critical IoT applications, such as IoV [238]. Clustering techniques can contribute to QoS improvement in IoT systems through dividing nodes to groups and improving QoS for each group 
in a distributed manner. For example, in [212] the authors introduce a clustering based routing algorithm for IoT networks to reduce end-to-end delay. They reduce interference resulting in delay and balance the load among nodes in wireless mesh IoT networks. In the following, we discuss existing clusteringbased methods that deal with QoS requirements.

1) Reliability: By reliability, we mean the rate of data successfully received by the destination node in the network. Clustering can contribute to the reliability of the network by improving the stability of the network. Below, we discuss existing solutions in this context.

\section{Existing solutions and techniques include:}

- Network stability improvement: Clustering can improve the reliability of the IoT network by avoiding node death and maintaining connectivity [239]. As an example, in [170], the authors propose a method to reduce inter-cluster communication cost to improve reliability in data transmission by assigning more resources to data forwarding and less amount of resources to the transmission of network management data. The proposed method establishes a tree determining connections between two nodes based on a weighting method and detects more reliable and affordable routes for data transmission to the BS. In [240], the authors propose a clustering-based routing algorithm called REMI for RPLbased IoT networks to provide faster multicast dissemination of messages, resulting in reliability improvement. In [241], the authors introduce a new clustering technique based on quantum particle swarm optimization (QPSO) with the improved non-dominated sorting genetic algorithm (NSGAII). The clustering technique introduces a trade-off between QoS and energy consumption of Industrial IoT.

2) Delay: Optimizing the efficiency of clustering-routing techniques can reduce delay (i.e., end-to-end delay). Generally, there is a trade-off between energy consumption and delay [37].

\section{Existing solutions and techniques include:}

- Reducing number of hops: The optimal solution is sending data directly from each node to BS, causing high energy consumption. Therefore, a challenge in designing routing methods is how to reduce delay and improve energy consumption at the same time. In [242], the authors introduce a delay-aware clustering algorithm for data acquisition in heterogeneous WSN-based IoT networks. In the proposed approach, the nodes with higher radio range act as relay nodes to reduce the number of hops [242], [243]. In [52], [55], two fixed hops are employed in intra-cluster routing to reduce delay in clusters.

- Load balancing: Load balancing can optimize lengths of queues in middle nodes, resulting in less delay. Network congestion is a result of an unbalanced load in the network which can cause high latency. Unequal clusters can cause delay due to unbalanced data transmission in different areas of the network [164]. In addition, selecting $\mathrm{CHs}$ /forwarders with low amount of resources can further increase delay [244]. In [245], the authors introduce a treebased WSN clustering method which can balance the load of the network by reordering clusters based on available streams to reduce dynamic end-to-end delay. The proposed method analyzes the packet streams on-the-fly and changes the clusters and routes to reduce delay. In [185], Kumar et al. compare K-means, hierarchical clustering and fuzzy Cmeans clustering techniques to group IoT nodes. They use these techniques to design a response time-aware scheduling model for IoT to improve QoS and show that K-mean is more efficient compared to others regarding the prioritized message delivery model.

- Routing optimization: inter-cluster and intra-cluster routing can highly influence delay by determining shortest-fastest routes. As routing is not the main focus of clustering, different techniques are introduced to achieve concurrent routing in clustering techniques [17]. In [243], the authors establish clusters based on an improved K-Means algorithm. They split and merge clusters to restructure the network resulting in a non-uniform clustering structure. Then, they create a tree for data fusing based on unequal clusters to utilize time slot scheduling, resulting in delay reduction.

3) Throughput: It refers to the amount of data that is successfully transferred through the whole network for a certain period of time.

\section{Existing solutions and techniques include:}

- Network stability improvement: Infrastructure-independent networks are not always stable because of node mobility, node failure, unreliable connections, and node death due to energy depletion, to name the most important ones. By having a more stable infrastructure, the network can have more reliable routes to transfer data which improves throughput. Clustering serves as an efficient means to improve stability by reducing the time for network recovery in unstable network circumstances. Some clustering techniques (e.g., in [246]) increase the number of alive nodes as a solution to improve the stability of the network. In [247], the authors introduce a clustering technique for IoT networks to conserve energy during routing, resulting in less probability of node death and so improving stability. They improve stability of the network by reducing delay, packet drop and energy consumption, resulting in better throughput.

- Stable number of CHs: A certain number of CHs with stable communication between $\mathrm{CHs}$ and $\mathrm{BS}(\mathrm{s})$ can improve the throughput of the network and keep the network reliable. Having a deterministic number of $\mathrm{CHs}$, the rate of data delivery to the BS will be predictable and the network throughput will become stable accordingly. In [248], the authors improve the throughput of heterogeneous IoT networks by proposing a clustering technique that uses weighted election probabilities to select a stable number of $\mathrm{CHs}$ among resource-rich nodes.

- Data aggregation: Data compression and aggregation are two methods to optimize the volume of data to transfer. Using these methods, the volume of data received by BS(s) would be less than the accumulative data generated in all nodes which can improve throughput and decrease energy consumption. In [249], the authors introduce a method to eliminate repetitive readings of generated packets during intra-cluster and inter-cluster routing to reduce the volume 
of data to transfer.

4) Jitter: Another parameter that can improve QoS is jitter prevention. It is especially important for multimedia and realtime applications which should keep the connections stable and live, and also control network congestion [250].

\section{Existing solutions and techniques include:}

- Tree-based clustering: Tree-based clustering structures can balance and possibly predict the volume of data in each route which can reduce jitter. In [159], the authors introduce a hierarchical tree-based routing protocol for cluster establishment. They introduce a reactive mechanism that use the history of the network to form $\mathrm{CHs}$ and reduce jitter. In [251], the authors introduce an optimized clustering communication protocol based on intelligent computing. The proposed clustering is a tree based technique improves energy consumption, but also reduces jitter and delay based on a cost function for establishing the tree.

- Splitting data streams: Ad-hoc networks use some nodes as hop to transmit data where each hop can be shared among different streams. Different stream rates, within a hop, can affect each other by changing the priority of packets in the forwarding queue, leading to jitter creation. By splitting packet streams in middle nodes or using different middle nodes for different streams, the effect of streams on each other will be reduced, e.g., effect of a multimedia stream on a temperature sensor stream which can be delay, jitter, or low throughput. In [206], the authors introduce a clustering technique for multimedia IoT networks to divide the large multimedia sessions into simple sessions based on the network situation to reduce jitter.

IoT-specific concerns. As discussed above, there are some clustering-based solutions addressing QoS requirements in IoT, however tackling the QoS issues of IoT systems by clustering introduces the following challenges:

- Quality of Experience (QoE): QoE is a novel concept which should be supported by IoT networks. QoE is aimed to improve the QoE of IoT users by receiving online feedback from them and the network infrastructure based on avoiding and solving network problems [252]. In other words, applications which interact with a human need to improve QoE, however supporting QoE can influence applications that do not interact with a human but have critical QoS requirements. Since no work exists on supporting QoE through clustering techniques in WSNs [19], it becomes challenging to address QoE in clustering techniques introduced for IoT.

- Applications requirements: Many IoT applications demand for critical QoS requirements. In special cases like IoV that deal with safety, QoS must be supported and it is not an optional parameter in improving the efficiency of the network. Since an ad-hoc IoT network may host multiple applications with various QoS requirements, the middle nodes in clustering will face a challenge on how to meet the QoS requirements in those applications that may be in conflict.

\section{E. Network Management}

This is an important challenge in IoT networks due to their large scale of deployments, and heterogeneity and mobility of nodes. In IoT applications, networks are more dynamic compared to WSNs, e.g., IoV and smart cities, making network management difficult. To manage the IoT network, dividing the network into geographical partitions and assigning the responsibility of partition management to local nodes is the primary solution. As network partitioning is the main task of clustering, different aspects of network topology management are satisfied by clustering techniques as listed below:

1) Scalability: Due to having many nodes such as sensors, wearables, user equipment, etc., IoT networks have to be scalable and connect thousands or millions of nodes.

\section{Existing solutions and techniques include:}

- Distributed management: Centralized network management solutions are not efficient in large-scale networks and impose high overhead. The authors in [253] introduce a scalable MQTT model in clustered IoT networks. Each cluster has some nodes as MQTT message brokers. They are used as a backend system to service MQTT clients and balance the load of the network in massive scale IoT networks. The authors in [254] introduce a method to search IoT services relying on a hierarchical network of semantic gateways. They use a recursive agglomerative clustering technique to provide a routing table of semantic gateways. It establishes clusters based on characterizing dissimilarities between services and distances. The authors in [199] propose a method to divide the regions where sensors are deployed into a grid and use a clustering index tree to manage and establish these grid cells.

- Reducing communication overhead: Communication overhead is another challenge in supporting scalability. In [130], the authors design a clustering method that reduces communication overhead to support scalability. Neamatollahi et al. [162] introduce a clustering method that supports scalability by applying an energy-efficient distributed algorithm for all levels of clusters to reduce communication overhead based on the number of nodes.

2) Stability: Stability is one of the management objectives which can make the network infrastructure more reliable in terms of connectivity, QoS, and fault tolerance. Different challenges can affect the stability of a MANET like mobility, node failure, etc. The most important existing solution used by clustering techniques to provide stability is:

- Avoiding node death: Clustering techniques reduce energy consumption of nodes to avoid node death and keep the routes and the cluster more stable. ACHTH-LEACH [89] is a method which provides reliable routes and a more stable network infrastructure to transfer data by selecting resource-rich nodes as $\mathrm{CHs}$. In this method, routes are established for a long time and nodes can trust routes to be fully connected during their data transmission. In [255], the authors introduce a hierarchical clustering algorithm for dynamic and heterogeneous IoT. This algorithm considers battery lifetime of the $\mathrm{CHs}$ to improve lifetime of the network and consequently make the network more stable. 
3) Mobility management: Mobility has always been considered a big challenge in wireless networks. Although mobility is an advantage for many applications, it can introduce various challenges in the network such as connectivity, reliability, and stability.

\section{Existing solutions and techniques include:}

- Inter-cluster handover mechanisms: Mobility of nodes is supported by clustering based on handover mechanisms among clusters. Mobile nodes may join and leave the clusters during the network lifetime. Some methods are introduced to support mobile $\mathrm{CHs}$ and BSs for information gathering in the network [154] [163]. In [143], CHs are considered mobile and used as mules to gather and transfer data. In [105], the authors use the speed of each node's movement as a parameter to select CHs. By using TDMA to schedule access to the medium in each cluster, $\mathrm{CHs}$ estimate the connection time between members and $\mathrm{CHs}$ to detect the mobility of nodes between clusters. In [256], the authors introduce a method to enable reliable uplink communications for clustered UAV IoT devices considering the energy consumption. They show that the proposed approach, can manage mobile IoT nodes as well as provide reliable uplink communications. In [257], the authors introduce a clustering technique for smart cities to optimize the planning of the transportation traffic network among hotel service centers in Tianjin. They introduce a clustering algorithm using the deep belief network (DBN) to divide regions and determine their service centers in Tianjin. In [258], Jabeur et al. introduce a new firefly-based clustering algorithm in which real-world things (RWTs) organize themselves into clusters during the macro clustering phase and integrate small neighboring clusters. Clusters can ask RWTs to join or leave the cluster due to events and their impacts.

IoT-specific concerns. As mentioned above, there are some clustering-based solutions addressing topology management (i.e., scalability, stability and mobility) in IoT. However, addressing topology management in IoT through clustering introduces the following challenges:

- Supporting QoS in scalable networks: As scalability is a critical challenge in large scale IoT systems (e.g., UDN networks, IoV, and smart cities), supporting QoS would be realized in a distributed manner in order to reduce the network management overhead [259]. In addition, two-way communication is considered as a challenge to support QoS, because in some cases QoS of a stream from a node to a destination is different compared to the QoS of the responses from the same destination to the same node.

- Highly dynamic applications of IoT: Mobility is a common aspect of several IoT applications such as IoV and smart cities. Adopting clustering as the mobility handling solution can impose high overhead when the network undertakes reclustering due to the high mobility of nodes.

\section{F. Network Connectivity}

Clustering can improve connectivity as each node has at least one connection to other nodes (directly) and also to BS (directly or indirectly). There are two aspects of connectivity that clustering can improve as listed below.

1) Improving coverage: A better coverage can connect more nodes to the network and extend the sensing coverage area.

\section{Existing solutions and techniques include:}

- Radio range management: In some clustering techniques, there are specific policies to limit radio range of nodes to improve coverage. In [115], the authors use a directional antenna to reduce energy consumption and also coverage of the network by controlling the transmission range of directional antennas. In [260], the authors introduce a model to analyze clustered D2D IoT networks in three-dimensional spaces based on Thomas Cluster Process (TCP). They evaluate the network in the presence of co-channel interference from both the same cluster and the other clusters, and investigate the coverage probability.

2) Improving nodes connectivity: A good connectivity among nodes can make the network infrastructure reliable and fully connected resulting in improving data delivery.

\section{Existing solutions and techniques include:}

- Establishing stable connections: In mobile networks, connectivity is a big challenge because mobile nodes can loose their connection dynamically. In [105], the authors introduce a method for mobile WSNs to improve connectivity which is based on an estimated connection time between $\mathrm{CHs}$ and members. If connections between members and a $\mathrm{CH}$ are lost, the members detect connection loss based on the estimated connection time and broadcast a request to find a new $\mathrm{CH}$ to avoid packet loss. Clusters overlapping can improve connectivity of nodes and coverage. However, high overlapping can cause various problems, especially delay because of having more hops between a node and BS. In [148], the authors introduce a clustering technique that can control the overlapping of the clusters and reduce delay and energy consumption using a hierarchical infrastructure. In [255], the authors introduce a new clustering technique for heterogeneous IoT networks based on selecting the optimal number of $\mathrm{CHs}$ to improve connectivity of hierarchical communication between nodes and BS. In [261], the authors introduce a cluster-based framework for Unmanned Aerial Vehicle (UAV) IoT networks that is adaptive, selfconfigurable, and resilient. The proposed framework cognitively adapts with IoT network changes to provide reliable connectivity between spatially dispersed smart devices.

IoT-specific concerns. Connectivity is a challenge due to establishing the IoT network over an existing network infrastructure that is perhaps unreliable. Below, we discuss challenges in applying clustering techniques to tackle IoT connectivity concerns.

- Network interfaces: Each route in IoT can include nodes with different types of network interfaces and each network interface may have various disadvantages compromising the reliability of the whole route. As an example, a Zigbee connection between two nodes in a route can be less reliable and cause higher delay when all other connections of the route are $\mathrm{WiFi}$. In addition, although some nodes are 
physically close to each other, because of lacking a common type of network interface, they can not connect to each other and need middle nodes to connect.

- Network infrastructures: Some technologies like 5G can serve as the network infrastructure of future IoT systems, even though they still suffer from some connectivity problems, e.g., blind spaces and millimeter waves are absorbed by physical obstacles [262]. The characteristics of the underlying IoT network infrastructure can affect the performance of clustering techniques in connectivity provisioning.

\section{G. Data Delivery Improvement}

It can enhance QoS and reduce energy consumption and network overhead by reducing packet re-transmission, data loss, etc. There are a number of metrics for packet delivery that can be improved by clustering techniques as listed below:

1) Improving packet delivery ratio: It is a parameter that has a high correlation with throughput.

Existing solutions and techniques include:

- Improving reliability: This refers to data loss and node failure reduction, and connectivity improvement in the network. In [165], a method has been proposed to improve the packet delivery ratio by selecting $\mathrm{CHs}$ based on their remaining energy, QoS parameters and the location of nodes. The proposed method uses the immune-inspired optimization algorithm to select routes for delivering packets. Industrial IoT is used in [263] as the network infrastructure featuring a grid-based clustering algorithm to model the network load. The network is divided into unequal grids based on the optimal grid length to optimize energy consumption and improve the packet delivery ratio.

- Reducing data loss: Data loss can also cause serious problems, especially in safety critical applications, such as fire detection, disaster management, and military-based information. Clustering can be used to detect node failures and reduce data loss. In [126], Izadi et al. introduce a clustering method that can detect node failure in an acceptable time after failure, while avoiding data loss. In [247], the authors introduce a routing protocol for IoT networks based on clustering to maximize throughput and decrease packet drop ratio together with reducing energy consumption.

2) Optimizing number of packets received by $B S$ : which has a high correlation with packet delivery ratio, but it refers to the amount of data received by BS in a certain time period compared to the data generated in nodes. In some papers, this parameter has been investigated as a metric for evaluating throughput of the network [161] [21].

Existing solutions in WSNs and IoT. Clustering techniques can be leveraged to optimize the number of packets received in BS. In the following, we discuss them:

- Network lifetime improvement: Improving network lifetime can increase the number of packets received over time by BS [152] [158]. In [248], the authors introduce a clustering technique to improve the network lifetime and consequently the rate of packet transmission to BS.

- Data aggregation: In some clustering techniques, the number of packets sent to BS is reduced to improve QoS and reduce network load, and energy consumption. Data aggregation, compression and fusion are the solutions applied in $\mathrm{CHs}$ and middle nodes to reduce the volume of data [80]. In [264], the authors introduce a method to fuse data on clusters established based on RSSI of IoT nodes. Access points are the CHs, exploiting RSSI to establish clusters. The fused data is then sent to the Cloud.

IoT-specific concerns. Like WSNs, the packet delivery rate should be improved in IoT to make the network infrastructure more efficient. Below, we discuss the challenge in applying clustering techniques for packet delivery improvement in IoT:

- Unreliability and instability of network infrastructures: They are the challenges against packet delivery improvement in IoT. To reduce data loss and improve packet delivery ratio, node failure and connection failure should be reduced as discussed above. The stability of the IoT network is compromised due to high dynamicity. In some IoT applications like IoV and smart cities, it is very challenging to keep the network infrastructure stable compared to WSNs.

\section{H. Security}

There are different types of attacks that can happen due to ad-hoc characteristics, e.g., hole attacks and DDoS [265]. Different techniques have been introduced to solve the attacks and detect malicious nodes, especially hole attacks which are very common in ad-hoc networks. In [266], the authors analyse the security mechanisms in IoT networks based on clustering. Existing solutions and techniques include:

- Distributed trust-based models: Clustering techniques can enable distributed trust-based models. In such models, each group of nodes includes one or more nodes that is (are) trustworthy. The trustworthy nodes are responsible to evaluate the local nodes in case of security attacks. In [267], the authors introduce a trust-based $\mathrm{CH}$ selection model for WSN-based Intelligent Transportation Systems (ITS). Each $\mathrm{CH}$ is selected based on residual energy, trust value and the number of neighbors. Each node is responsible for monitoring the behavior of its neighbors. $\mathrm{CHs}$ are in charge of evaluating the trustworthiness of each member based on analyzing other members' evaluation. In [178], a clustering technique is introduced to provide security for wearable IoT devices. They propose a clustering-based anonymity method to preserve privacy of data gathered from wearable IoT devices and guarantee the usability of the collected data. In [268], the authors propose a centralized detection system based on the data gathered from clusters to detect the malicious gateways of clusters in IoT networks using packet drop probability as a means to monitor the gateways.

IoT-specific concerns. Security is one of the main concerns of IoT systems because of having weak and vulnerable nodes. Using clustering techniques for addressing security concerns in IoT will face a number of challenges as listed below:

- Highly dynamic IoT: The massive scale of IoT deployments and their high dynamicity can pose more security challenges. As unknown IoT devices can join the network 
in applications like smart cities, applying distributed trustbased models would be a challenge as trustworthy nodes may have not enough time to evaluate unknown nodes.

- Resource-constrained IoT devices: Security solutions for IoT are mostly focused on authentication and authorization. As supporting security is a heavy task, applying authentication and authorization techniques is resource demanding. In distributed models, clusters may include heterogeneous nodes, thereby having the same local authentication and authorization techniques among members and the $\mathrm{CH}$ would be challenging with respect to the heterogeneity of nodes.

\section{Physical Layer Support}

Although clustering techniques logically belong to the network layer, they can contribute to the efficiency requirements of the physical layer, e.g., improving the efficiency of bandwidth reservation methods. Existing approaches in this field revolve around distributed resource management.

\section{Existing solutions and techniques include:}

- Distributed resource management: Clustering techniques can group nodes with a local resource management mechanism in each cluster in order to more efficiently manage, schedule and share physical resources, e.g., medium and bandwidth allocation to avoid transmission interference. As a fundamental clustering method, adaptive clustering [54] is used to efficiently allocate bandwidth to members in each cluster. It uses a virtual circuit definition to assign real-time connections and reserve time slots in clusters. In [269], the authors introduce a classification technique to establish cells (i.e., clusters) for IoT architectures based on a mesh of IoT devices, roadside repositories and vehicular mobile gateways. The model uses routing trees, the network geometries, capacity of wireless connections, and stability of IoT queues to perform the classification. In [270], the authors introduce a method to utilize bandwidth allocation based on inter-cluster aggregation which reduces the volume of data in a hierarchical clustering technique in each layer, in addition to reducing energy consumption and bandwidth allocation in upper layers. In [245], the authors use a heuristic scheduling model in clusters to adapt the network with end-to-end delay and bandwidth allocation requirements. They use a scheduling model that can react to different data flow changes in routers $(\mathrm{CHs})$ by re-ordering active periods of clusters and optimizing the time-slot to access shared channels by each cluster. In [271], the authors introduce a multiple-input-single-output (CMISO) method to prolong the lifetime of $\mathrm{CHs}$. In the paper, they formulate the cooperative coalition selection for CMISO and use a quantum-inspired particle swarm optimization technique to select the optimum cooperative coalition.

IoT-specific concerns. Supporting physical layer requirements is an important issue in IoT. As listed below, different aspects should be considered for designing IoT clustering techniques to support physical layer objectives.

- Ultra Dense Networks (UDN): In several IoT applications, the network infrastructure is a UDN which needs to allocate medium to nodes efficiently, e.g., by using scheduling techniques. To reduce medium access interference in an area, nodes may reduce their radio ranges, which can compromise network connectivity. Thus, to establish clusters, reducing the interference needs to be considered during $\mathrm{CH}$ selection and joining of the members. Clusters that can not support UDN networks may not be a good choice for optimizing resource consumption. Densification is a new definition in D2D-enabled UDN cellular networks that resembles clustering, grouping and limiting D2D communication between nodes to reduce interference [272].

\section{J. Multi-Sink Support}

Supporting multi-sink is a great solution to improve the efficiency of the network by increasing the number of data routing options to the end node. It can improve throughput, packet delivery and fault tolerance, as well as reduce delay. Although multi-sink networks are more efficient compared to single-sink networks, clustering techniques which are able to support multi-sink networks are more complex to design.

\section{Existing solutions and techniques include:}

- Multi-point to multi-point (MP2MP) clustering-routing: As the main solution, clustering-routing techniques need to connect nodes to the best sink (e.g., with respect to resource consumption and delay) as an optimization problem. Establishing these connections needs to have a multi-point to multi-point inter-cluster routing. In [154], the authors propose a method to support multiple mobile sinks. It uses $\mathrm{CHs}$ to maintain optimal routes which have minimum hops to the latest location of mobile sinks. In [68], the authors introduce a clustering technique that can support multiple mobile sinks to gather data based on queries. They improve the 'success rate' which denotes the ratio of the number of successfully received data packets at BSs to the total number of data packets generated by a node. In [273], the authors introduce an SDN-based solution for resource-constrained IoT devices that need to select the best router to connect as the edge node. The network includes multi-sink (i.e., edge nodes) and the clusters use RPL to establish routes between clusters and edge nodes. They consider different metrics and objective functions for routing, including Minimum Rank with Hysteresis Function (MRHOF) and Zero (OF0). In [274], the authors propose a Shortcut Addition strategy based on the Particle Swarm algorithm for multi-sink IoT networks. It constructs network topology based on a smallworld network. A fitness function is created by combining the average path length and load of the sink node to evaluate the results of the particle swarm optimization (PSO) technique. Each node selects the sink closest to itself based on the results of the PSO algorithm. Sung et al. in [275] introduce a multihop clustering technique to minimize the required Internet connection in an IoT network. A node per cluster is responsible for connecting the nodes to the Internet and the clustering technique minimizes the distance between the end-nodes and those connected to the Internet.

IoT-specific concerns. Although supporting multi-sink is an important factor in improving efficiency, some issues should 
be considered in designing an IoT clustering technique that supports multi-sink. We discuss the most important one below:

- Selecting the best sink (gateway): Supporting multi-sink networks is an important objective that can increase the efficiency of ad-hoc networks in different aspects such as QoS and resource consumption. Most clustering techniques that support multi-sink focus on designing a routing method to find the best route to transfer data to outside of the network. Although some clustering techniques consider multi-sink, especially as a scenario in their performance evaluation, there are not many techniques that have a specific solution for multi-sink networks. In IoT, selecting the best sink is a multi-objective problem. Two steps are introduced to select the best sink: $i$ ) selecting the best sink based on different costs to transmit data through the sink; ii) selecting the best route from the node to the selected sink, which is a challenging issue in IoT as nodes in a route may be heterogeneous in terms of the properties of network interfaces (e.g., bandwidth and delay) [276].

\section{Iot Clustering AND Modern Networking PARADIGMS}

So far, we discussed how clustering can address various quality-related objectives in IoT. Along this, clustering can be exploited to facilitate adoption of recent technologies integrated with IoT systems, in particular with respect to the barriers originating from networking issues. We focus on four new pioneering computing and networking paradigms that are being rapidly integrated with IoT, namely Blockchain, SDN/NFV, Fog/Edge, and 5G. First, we describe each of them briefly, especially from the networking viewpoint. Next, we discuss how clustering can be exploited to improve various design aspects in these technologies. For each technology, we discuss the limited number of existing clustering-based approaches. Then, the benefits of clustering is presented per technology. Finally, we identify the challenges raising in integration of clustering techniques with these technologies.

\section{A. Blockchain and IoT}

Blockchain (BC) technology is fundamentally developed to store transactions reliably in a distributed manner, especially for cryptocurrencies. BC is a distributed ledger among network nodes to store transactions and improve the security, reliability and efficiency of a decentralized network. Consensus algorithms [277] are used to guarantee that the $\mathrm{BC}$ is decentralized, reliable and secure. In a BC network, all nodes have $\mathrm{P} 2 \mathrm{P}$ connections similar to BitTorrent and contribute to storing, mining, and accessing transactions. Each node has two keys, private key and public key, to encrypt and decrypt the messages from and to the node, respectively.

$\mathrm{BC}$ can be used in various security-related applications, not only cryptocurrencies. Concerning IoT applications, security and privacy are still two major challenges due to the scale and distributed nature of IoT, and the resource scarcity of IoT nodes. Traditional security methods consume a lot of energy and impose high processing overhead. Integrating IoT and $\mathrm{BC}$ can promise a network infrastructure that becomes safe and secure by storing transaction records on network nodes in a distributed manner. General IoT security methods are not distributed and need many-to-one traffic streams which cause vulnerabilities such as central node failure, delay, and unnecessary energy consumption [265], [278].

Clustering techniques can facilitate integrating IoT and BC to improve the security and efficiency of IoT. Using clustering techniques, nodes can share their resources in a distributed management model to perform resource consuming tasks of BC. In addition, grouping nodes can help BC to provide smaller logical networks that can share resources or work based on trustworthiness. Mining as a complicated task should be performed by several nodes and clustering techniques can enable distributed execution of it.

Common features of IoT and BC make BC suitable to address the security needs of IoT as listed below:

- Both technologies are decentralized which can support scalability and eliminate non-efficient traffic overheads.

- BC provides security and privacy on top of the overlay network which includes untrusted nodes. Generally, IoT nodes are resource-constrained, while evaluating their trustworthy level is a resource consuming task.

- $\mathrm{BC}$ nodes are anonymous due to supporting privacy of users which is suitable in IoT use cases.

Although BC can offer many advantages for IoT systems, there are significant challenges which should be addressed to implement $\mathrm{BC}$ as listed below:

- BC technology uses some nodes in the network as miners to mine the blocks of the ledger. Mining is a highly resourceintensive task that calculates Proof of Work (POW) as a cryptographic puzzle to add new blocks to BC. Each verified transaction will be added to a pending block in multiple miners and all of them should mine the same block, causing delay, resource consumption, etc. Since IoT nodes are generally resource-constrained, mining is the main challenge in implementing $\mathrm{BC}$ in IoT due to resource consumption, delay and management of distributed mining on different machines.

- BC techniques can be categorized as public and private and also permission-less and permissioned. Public BCs can have global-anonymous participants, but private BCs are application-specific and designed to establish a BC among known participants, mainly to reach a specific objective. On the other hand, participants of permission-less BC do not require permission to be part of the $\mathrm{BC}$, but in permissioned $\mathrm{BCs}$, a control layer controls the actions performed by permissioned participants.

- BC has high overhead traffic which is not suitable for IoT devices that suffer from bandwidth limitation.

Recently, some works have been carried out to apply BC in IoT, out of which a number of approaches use clustering techniques to integrate IoT and BC. In [279], the authors propose a method based on the concept of 'The friend of my friend is my friend'. They use BC for WSN-IoT mobile networks to solve authentication problems of resource-constrained nodes in IoT. The proposed method authenticates mobile nodes that are moving from one cluster to another. They use $\mathrm{BC}$ to reduce the 
number of exchanged messages to provide security and also eliminate Key Derivation processing. She et al. [280] propose a $\mathrm{BC}$ trust model in 3D environments to detect malicious nodes in WSNs based on Consortium Blockchain and clustering techniques. BS publishes contracts and $\mathrm{CHs}$ use them as verification nodes and also provide digital certificate-based identity information for cluster members. In the proposed method, $\mathrm{CHs}$ are pre-selected to detect malicious nodes. In [281], the authors introduce a security layer in IoT to detect and isolate malicious nodes based on clustering techniques. They consider $\mathrm{CHs}$ as resource-rich nodes which are simultaneously miners and also keep a copy of nodes' firmware. They assume that clusters are already available and if the $\mathrm{CH}$ is compromised, the whole cluster would be untrustworthy. They use an authentication service as a smart contract on $\mathrm{CHs}$ to authenticate nodes. For authorization, all nodes in a trusted cluster should be able to authorize other members.

In [282], the authors proposed a clustering technique for IoV to reduce the load of transactions on IoV devices. The proposed method determines slots which can update BC ledgers optimally by selecting appropriate $\mathrm{CHs}$. Using the clustering technique, they reduce energy consumption for about $40 \%$ and the volume of transactions for about $82 \%$. A distributed ledger mechanism is used among CHs and it makes the decision about when and how the ledger should be updated on CHs. They propose a location-based ledger-offloading model that selects new $\mathrm{CHs}$ to optimize energy consumption.

In [277], the authors use a BC model to provide a distributed cyber infrastructure for the future smart grid systems. They proposed a model based on public and private keys in which each meter-node in the network collects data, encrypts it and then broadcasts it. Other meter node decrypts data and verifies the data by a voting method. If the data has enough votes to verify, then the data is packaged into a block. Their application is a smart meter data aggregation network consisting of regional BC and wide-area BC. In this application, smart meters are clustered called regional clusters. Then, clusters establish regional $\mathrm{BC}$ networks which create a wide-area $\mathrm{BC}$ network. The model establishes a two-tier hierarchical $\mathrm{BC}$ where $\mathrm{CHs}$ as gateways secure and maintain the wide-area BC network. A $\mathrm{CH}$ can be a storage or processor of the data sent from cluster members.

The authors in [283] use BC in IoV networks to improve the privacy and security of vehicles. They propose a decentralized architecture for the smart vehicle eco-system in which each node is part of an overlay network and a cluster. $\mathrm{CHs}$ manage $\mathrm{BC}$ and maintain the architecture called overlay block managers (OBM). CHs use changeable public keys and multisig (multi-signature) transactions. Multisig transactions are broadcast among OBMs. Each node of OBM has a list of public key pairs to verify signatures. Each OBM node controls access to the transactions of its cluster members.

Ali Dorri et al. [284] propose a new BC method called Lightweight Scalable Blockchain (LSB) which replaces the mining procedure with a new trustworthy model in a clustered infrastructure. In [285] and [286], A. Dorri et al. propose a method based on LSB to replace the mining model with a trustworthy mechanism in smart homes. $\mathrm{CHs}$ are used as nodes that can trust each other and store blocks, ledgers and transactions. By having clusters, members can trust $\mathrm{CHs}$ and $\mathrm{CHs}$ can trust each other using multi-signature transactions. Moreover, scalability can be supported by allowing forking in different clusters or different layers of hierarchical clustering techniques. In [287], the authors propose a secure routing protocol for smart homes. They use a clustering technique to separate indoor spaces by allocating region numbers for each space like kitchen, bedroom, etc. They create super clusters and common clusters. Super clusters include reference nodes that can connect to a common cluster of $\mathrm{CHs}$ directly. They use a beta-based trust management model to improve the security of $\mathrm{CH}$ selection.

In the case of permissioned BC, Amiri et al. [288] introduce a cluster-based sharding technique for permissioned $\mathrm{BC}$ to support scalability of BC. Their proposed technique, called SharPer, establishes clusters to assign different shards of data to the clusters. Each data shard is replicated on cluster members to support intra-shard, and cross-shard transactions. Their proposed $\mathrm{BC}$ ledger is based on the directed acyclic graph and establishes consensus by clusters for cross-shared transactions based on a flattened protocol. Hyperledger fabric is also introduced by IBM as a modular and permissioned architecture to assign the roles to nodes in a distributed architecture of $\mathrm{BC}$ [289]. In [290], the authors propose a BC-based multi-layer security model for IoT. They divide the IoT network to kunknown clusters based on Genetic algorithms and the PSO algorithm. In each $\mathrm{CH}$, they provide a local authentication and authorization technique to improve the security of IoT. They also use $\mathrm{BC}$ to provide security for inter- $\mathrm{CH}$ communication and $\mathrm{CH}$-to-BS communications. They also propose a global $\mathrm{BC}$ to secure the BS communications. An open source BC hyperledger fabric is used to verify the techniques. In addition, Mbarek et al. in [291] introduce a multil-evel BC system to improve data security and privacy of IoT along with reducing the response time and resource consumption. They propose three levels called mirco level, meso level, and macro level, which contain members of clusters, $\mathrm{CHs}$, and servers of $\mathrm{BC}$, respectively. In addition, the authors introduce the use of mobile IoT devices that move in different levels of IoT and $\mathrm{BC}$ to perform $\mathrm{BC}$ functions, e.g., hashing and encryption.

Additionally, there exist some $\mathrm{BC}$ research works that use clustering methods, while their main contribution is not proposing new clustering techniques. In [292], the authors use $\mathrm{CHs}$ to collect, fuse and encrypt data gathered from nodes. They use attribute-based encryption to provide confidentiality and access control. CHs are the owners of data and BC miners are the other nodes. In [293], the authors use clustering as a node grouping technique in the supply chain. $\mathrm{CH}$ s are selected randomly, proposing a new block when needed.

1) Importance of clustering for $B C$ : As mentioned above, IoT has some limitations which make implementing $\mathrm{BC}$ for IoT systems challenging. Clustering can be used to overcome these obstacles.

- Improve the compatibility of BC with IoT: As mining is a resource-intensive process, it can be replaced with other techniques, e.g., trustworthy mechanisms. Using clustering methods, the network can be structured in such a way 
that clusters trust each other and do not need to mine blocks. As explained before, A. Dorri et al. [284] propose a trustworthy mechanism rather than mining. Clustering can provide an extra overlay network on top of IoT, which can be used to group nodes. Grouped nodes can evaluate their trustworthiness and detect malicious nodes. Then, clusters can trust each other or have an extra layer that uses BC as well. In other words, by using clustering methods, it is possible to have a hierarchical $\mathrm{BC}$ which distributes responsibilities, not among nodes, rather among groups of nodes.

- Resource sharing in BC: In implementing a $\mathrm{BC}$, nodes need large amount of resources to mine blocks which are not applicable to IoT systems due to resource scarcity. Although mining is a centralized task, it is possible to perform it in a distributed manner. There exist two different approaches on distributed mining. Distributed miners [294] is the first approach which divides the mining task into work units and uses different machines to execute them. Bitcoin Plus, Bitp.it and Stealthcoin are examples of distributed miners. The second approach is called pooled mining [295], in which miners share their processing power to mine and split the reward based on the amount of their contribution. Both these approaches can be used in IoT for distributed mining based on share resources. Clustering techniques can be used to propose clusters as pools and $\mathrm{CHs}$ will serve as a coordinator among pools. Especially in Fog computing models, which have powerful nodes, it is possible to establish clusters to share resources of powerful fog nodes. On the other hand, Some BC solutions use solo mining [296] in which each miner includes only one node. In this case, clustering can determine and use $\mathrm{CHs}$ as miners. In this case, $\mathrm{CH}$ is responsible for mining transactions related to its members or neighbors. Besides, there are different $\mathrm{P} 2 \mathrm{P}$ based mining methods to increase the efficiency of BC which can be implemented using clustering. As an example, P2POOL [297] uses a peer-to-peer network of node miners to create a mining pool. Using clustering, a more efficient $\mathrm{P} 2 \mathrm{P}$ distributed network can be established to implement such methods.

2) Challenges of integrating clustering techniques with $B C$ : To integrate clustering techniques and $\mathrm{BC}$, we face a number of challenges as listed below:

- Heterogeneity: In resource sharing techniques, fairness is an important factor due to the resource-intensiveness of mining. Generally, IoT nodes are heterogeneous and the positions of nodes in IoT are unbalanced, causing unfairness. To design efficient clustering techniques, clusters should be equal in terms of available resources. Resource consumption of the mining process is generally unpredictable which may cause unbalanced resource consumption on different machines. To support fairness, all clusters should have balanced resources; thereby, clusters should be composed of heterogeneous nodes.

- Dynamicity: To execute tasks on shared resources in a cluster (i.e., mining and storing ledger), the cluster should be stable between two set-up phases. Mobility and dynamicity are two challenges, which make resource sharing difficult. Dynamic clustering is needed when a cluster is subject to dynamic changes. Additionally, using task offloading methods for mobile nodes can address the dynamicity problem.

- Compatibility: As mentioned above, standard BC is not applicable in IoT due to resource-intensive processing tasks in BC. Designing a method that can make the standard BC compatible with IoT can be useful, e.g., the technique proposed in [285]. However, such techniques like trustworthy based models essentially impose high traffic overhead rather than processing overhead. To have compatible models and eliminate mining, distributed techniques should be applied regarding control of their overheads.

- Scalability: Although different techniques can support scalability in BC IoT infrastructures, managing a huge number of nodes can induce high processing and traffic overheads. In large-scale networks, maintaining the efficiency of clustering would be a resource-intensive task.

\section{B. SDN, NFV and IoT}

Software-Defined Networking (SDN) is basically a centralized networking paradigm to decouple the control plane from the data plane of data transmission [298]. In SDN, the network intelligence (i.e., the control function or the control plane) is centralized at one or a set of control entities (i.e., SDN controllers), while the data forwarding plane is abstracted for applications and networks services. In the first generation of SDN, the OpenFlow protocol [299] is used between the control and data planes. For example, an OpenFlow switch has one or more forwarding tables that are controlled by a centralized controller, which realizes programmability in the control plane.

Network Function Virtualization (NFV) is the concept of relocating network functions from dedicated hardware appliances to software-based appliances running in the cloud environment or on general-purpose commodity equipment [300]. Using NFV, conventional network functions (NF) will run on a virtual machine (VM) as a one-to-one mapping model or is decomposed into smaller components called Virtual Network Function components running on multiple VMs. Virtual Network Functions (VNF), which represents the implementation of NFs, are deployed and executed on an NFV Infrastructure (NFVI). The NFVI consists of virtual resources, which are abstracted and logically partitioned from underlying hardware resources (computing, storage, and networking) through a virtualization layer.

1) Importance of clustering for SDN and NFV: Clustering has the potential to contribute to the efficiency of SDN and NFV, such as communication and control. In particular, clustering can enable a better division of network nodes and their association with the SDN control domain based on predefined communication criteria, e.g., nodes vicinity. Clustering can also enable more efficient implementation of the control component in SDN by facilitating distributed control through clustered nodes and their CHs. The main advantage of using clustering techniques in NFV is that the amount of traffic that needs to be traversed through NFV based nodes in the same cluster do not need to leave the cluster. This can significantly 
reduce the amount of traffic routed throughout the network, in particular in mobile edge computing networks.

However, there are not many works focusing on the exploitation of clustering techniques in SDN scenarios. In [301], as one of the few works in this category, a new clustering algorithm, The Whale Optimisation Algorithm (WOA) is proposed based on the concept of SDN for IoT systems. WOA considers both sensor resource restrictions and the random diversification of IoT node density in the geographical area. It divides the sensing area by the SDN controller into virtual zones (VZs) to balance the number of $\mathrm{CHs}$ according to the node density in each VZ. Then, it uses WOA to define the optimal set of $\mathrm{CHs}$ based on residual energy, communication cost and node density. In [302], scalability, fault tolerance and interoperability in the centralized control of the SDNs are challenged and a novel clustered distributed controller architecture is proposed in the real setting of SDNs. The distributed cluster implementation comprises of multiple SDN controllers. The clustered controllers perform load balancing by distributing the number of connected OpenFlow switches between instances of the controllers in the cluster. Similarly, in [303] a system is proposed to assign controllers to clusters by optimizing the maximal distance between two controllers in the same clusters in order to reduce the time complexity of load balancing in the SDN control plane. In [304], a model based on SDN is proposed to prevent different attacks in IoT systems. Clusters of SDN controllers are created and a $\mathrm{CH}$ selection process is proposed to manage and control different security issues in a particular domain. In [305], a WSN is divided into multiple clusters, with $\mathrm{CHs}$ acting as a controller in each network. Communication between nodes and $\mathrm{CHs}$ is managed by gateway nodes and each cluster is considered to be an SDN domain.

There is also a limited body of work on using clustering techniques in NFV. In [306], the concept of clustered NFV is proposed. An existing solution for combining clustering and NFV is leveraged to compute the optimal number of clusters to minimize the end-to-end time of MEC services. The proposed scheme is applied to form multiple MEC clusters of NFV enabled nodes within the radio access networks (RANs). In [307], the authors propose a systematic virtual networking architecture to perform the virtualization control and monitoring of a CPS, in which NFV configuration and orchestration can be realized. A game-theoretic topology decision approach is proposed to control the topology of the clustering and virtual network functions deployment at run-time in a CPS.

2) Challenges of integrating clustering techniques with $S D N$ and NFV: We identify the following future directions in using clustering techniques in SDN and NFV:

- Distributed control: thanks to the structure of clusters and the possibility of $\mathrm{CH}$-level decision making, the centralized components of SDN can be decentralized in cluster based topologies in order to address more efficiently the capacity concerns in large IoT applications. This implies that the data forwarding plane is limited to the nodes in a cluster, while inter-cluster data forwarding is still a challenging issue.

- Clustering for orchestrating in NFV: a key function of NFV is the realization of orchestrated virtual network functions, which can be implemented in different ways, like data flows. Clustering can facilitate the development of orchestration for NFV by utilizing $\mathrm{CHs}$ as the point of orchestration.

- Software-based clustering: a cluster is generally built on a network topology which adheres to the given clustering requirements. From the NFV viewpoint, a given clustering mechanism and the associated algorithm can be derived as virtualized functions installed on, e.g., a general-purpose computing device. In this way, more flexibility and dynamicity will be enabled in the implementation of clustering functions.

\section{Fog and Edge}

IoT devices and services are proliferating into a massive scale, thereby, IoT data, services and applications are being migrated to the Cloud, enabling powerful processing and sharing of IoT data beyond the capability of individual things. Moreover, due to requirements such as mobility support, location-awareness and low latency, the cloud computing paradigm has been recently extended from the core of the network to the edge-Fog Computing. The fog nodes, residing between the IoT devices and the Cloud can be structured in various forms, such as hierarchical topologies. More efficient organization of fog devices can lead to more efficient data collection, processing and transmission to the Cloud. Fog computing and edge computing are quite similar in meaning. Both are concerned with leveraging the computing capabilities in a local network to perform computation tasks that would otherwise have been executed in the Cloud. However, Edge computing usually occurs directly on the IoT devices or a gateway device that is physically close to the sensors and IoT devices. Fog computing moves the edge computing activities to computing nodes that are connected to the LAN or into the LAN hardware itself so they may be physically more distant from the sensors and IoT devices and spread geographically from edge devices towards the Cloud.

1) Importance of clustering in Fog and Edge: Clustering has the potential to address efficient data collection and processing in Fog- and Edge-computing environments. Clustering can not only improve the robustness of the Fog network but also allow better control of data management. As an example for the latter, IoT data processing in large deployments is bounded to a geographically defined area. Figure 5 shows an overview of how clustering can be applied to fog-based scenarios in which fog nodes are organized in a hierarchical model. This model is suitable for large-scale IoT deployments, e.g., in smart cities [308]. As can be seen in the figure, a cluster can be composed of a set of IoT devices and the associated fog node as the $\mathrm{CH}$. The nodes in a cluster can communicate through $\mathrm{D} 2 \mathrm{D}$ or $\mathrm{M} 2 \mathrm{M}$ technologies. In this model, the fog node is often called as the edge node acting as $\mathrm{CH}$. As the alternative scenario, a cluster can also consist of only fog nodes, which fits in scenarios with, e.g., fog-level load balancing and workload distribution for intensive data processing [309].

Recently, a number of works have been reported on the usage of clustering techniques in fog computing scenarios. The 


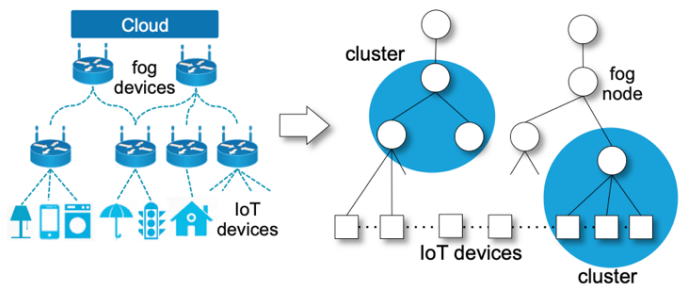

Fig. 5. A clustering model in hierarchical fog architectural models

more popular line of research in this area is related to using clustering for cost-efficiency, low power consumption, and robustness in fog networks. A comprehensive cluster-based approach in fog computing is proposed in [310]. It proposes an algorithm for cluster formation and load balancing for fog computing. The algorithm has a customizable design where metrics, scheduling rules, and clustering objectives can be set according to specific application and network requirements. Another application of clustering is in data caching. In [311], a clustering method is proposed to group spatially proximate user devices with mutual task popularity interests with their serving cloudlets. Then, cloudlets can cache the popular tasks' computations of their cluster members to minimize computing latency. In [312], a communication service between the fog layer and the IoT device layer in fog computing is provided, in which dynamic IoT device clustering is proposed to reduce the system complexity and the delay for the IoT devices with better channel conditions. The concept of edge-managed clustering is proposed in [313] to supervise the operations among cooperating devices forming a cluster. The cluster manager is proposed running on an edge access point, providing both connectivity and fast management response to the attached IoT devices.

There are also contributions to the application of clustering techniques in fog-based systems, e.g., clustering for the purpose of better application software management. In [314], an architecture for edge cloud PaaS is presented for application and service orchestration to manage and orchestrate applications through containers. They use clustering to create a cluster of Raspberry Pi nodes (as fog nodes) to run computationally intensive software. In [315], a distributed consensus algorithm is proposed for decision making in service-oriented IoT. Clustering is exploited to partition the IoT network into multiple clusters for a local consensus. The local consensus can be achieved within each cluster and can then be used to make consensus decision in knowledge sharing and integration of functional capabilities. The authors use a distance-based static clustering scheme for node clustering. In [49], the authors investigate container-oriented operational frameworks for IoT. They propose edge-managed clustering, where a manager supervises the operations among cooperating devices. The cluster manager runs on an edge access point to allow connectivity and fast management response to the attached IoT devices.

2) Challenges of integrating clustering techniques with Fog and Edge: We identify the following future directions in using clustering techniques in fog computing:

- Clustering for addressing privacy: clustering can be applied for fog devices that are in charge of collecting and processing privacy-sensitive data, e.g., the power consumption profile of a neighborhood. Using clustering, the collaborative fog nodes (for data processing) can form clusters with welldefined privacy constraints. In this way, the privacy issue will be easier to maintain, in addition to the fact that intercluster communications can be mapped to the privacy levels of the end-user application.

- Optimization of resource allocation: fog resource allocation is a complex problem. It refers to how the available fog resources should be allocated for data processing to satisfy quality requirements such as low latency, and cost. To allocate resources efficiently, optimization algorithms should be leveraged, taking into account the pre-defined optimization parameters in resource allocation. Clustering can be the basis for optimization such that the nodes in the cluster are identified based on the optimization goals [316]. In this way, the management of resources and execution of optimization algorithms will become more efficient.

- Clustering for running data-intensive tasks: in data-intensive applications, tasks may run on different nodes, while the results of task execution are sent to other nodes in the network. Clustering can serve as a local network of fog nodes hosting the execution of interrelated tasks and sending the task execution results to other nodes in the network, e.g., to the Cloud.

- Computation offloading: This is an important feature in fog computing due to QoS requirements or resource limitations. A task may be offloaded to a fog device from other nodes or the Cloud, or uploaded to the Cloud from a fog node, respectively. With respect to the former, a critical efficiency parameter of the offloading process is selecting the best candidate for task offloading. Clustering can provide a suitable platform for facilitating the selection process. In particular, when $\mathrm{CH}$ has an overview of the resource capabilities of its nodes, it can be exploited to find the node(s) for offloading based on the resource availability information. Moreover, when the computation offloading is limited to a geographical area (e.g., due to privacy preservation), the counterpart cluster(s) specify the boundaries for the techniques that should select the candidate nodes for offloading.

\section{D. $5 G$ and IoT}

Today, different generations of cellular networks cover at least $90 \%$ of the world's population. $2 \mathrm{G}$ providing voice communication, covers $90 \%$, while $3 \mathrm{G}$ used for transferring data and voice covers $65 \%$ of the people in the world [317]. 4G and Long Term Evolution (LTE) are the generations after $3 \mathrm{G}$ supporting IoT applications not only themselves but also by establishing concrete overlay network infrastructures like NB-IoT [317]. There are several issues in supporting IoT applications in the 4G network which need to be addressed, such as capacity, data rate, end-to-end delay, massive device connectivity, communication cost and QoE [318]. 5G is an emerging generation of cellular networks designed to enhance the efficiency of data transmission and address the problems of LTE. Although 5G is not designed specifically for IoT, it 
is expected that it will provide the main backhaul of IoT in 2025 [319]. A large number of solutions have been proposed on the integration of IoT applications and 5G infrastructures [320]. Third Generation Partnership Project (3GPP) as a collaboration among different associations designs standards for cellular networks like $5 \mathrm{G}$ to establish concrete infrastructures that support applications with requirements such as low latency, QoS, energy efficiency, etc. [320] [321] [322] [323]. Different technologies are emerging to improve the efficiency of $5 \mathrm{G}$, and different standards are being released based on them. Although there exists a large number of such technologies, some technologies are the foundation stones of $5 \mathrm{G}$. There are 5+1 fundamental technologies which form 5G [324] [325] [326] [327], including Millimeter Waves (mmWave) [328], Small Cell [322], Massive MIMO [328], Beamforming [329], Full Duplex [330], and Device-to-Device Communication (D2D) [331].

Although D2D is not a fundamental technology for $5 \mathrm{G}$, it is emerging as a significant technology to support $5 \mathrm{G}$ objectives. The above technologies can improve the efficiency of LTE and $4 \mathrm{G}$ to satisfy the requirements of $5 \mathrm{G}$. Although all these technologies support each other, Small Cell and D2D are used to form the network infrastructure, logically in the network layer. Almost all other technologies contribute to improvements in the MAC and physical layers. Small Cell is a technology that establishes a hierarchical network infrastructure to cover blind spaces, optimize energy consumption based on reducing the distance between devices and small cell BSs, and support QoS. Small cells break up a cell site into smaller cells, usable in indoor and outdoor environments called Macro cells, Microcells, Picocells and Femtocells, respectively, as listed in Table V. As an example, a Femtocell as the smallest cell can connect to a bigger cell or directly connect to the Internet by wire. The way of connecting is same for all types of cells. This hierarchical model can help 5G to cover all blind spaces, reduce energy consumption, and improve QoS [332].

Although Small Cell can enhance the efficiency of the network infrastructure management in $5 \mathrm{G}$, there are still some challenges with respect to supporting QoS, improving bandwidth and energy consumption. D2D communication can address these challenges. D2D is introduced for short-range communication between two devices without using BS, resulting in less energy consumption, and improving load balancing and QoS. Resource-constrained IoT devices normally do not support wide coverage of connectivity. D2D technology was first introduced by Qualcomm, called flashlinQ, to enable D2D in LTE-A [333]. It is officially addressed in 3GPP LTE release 12 [334]. D2D is introduced by 3GPP, also known as Proximity Service (ProSe) which supports multi-hop relay networks [335]. In release 15, the main objective is to improve the power efficiency of remote User Equipment (UE), such as IoT devices and wearables, by allowing them to establish D2D communication with UEs acting as a relay. Although D2D in ISM bands like Wi-Fi direct is well-established, D2D standardization in cellular networks is still a challenge.

D2D communication in $5 \mathrm{G}$ can enable the development of novel applications and enhance existing mobile applications. For example, social networks apps and games can use D2D to
TABLE V

THE SPECIFICATION AND COVERAGE AREA OF DIFFERENT 5G CELL TYPES

\begin{tabular}{|l|l|l|l|l|l|}
\hline Cell Type & Radius (Km) & Power (W) & Number of Users & Covered area & Example \\
\hline Macro Cell & 8 to 30 & 10 to $>50$ & $>2000$ & Outdoor & Cover a City \\
Micro Cell & 0.2 to 2.0 & 1 to 10 & 100 to 2000 & Indoor/Outdoor & Cover a Street \\
Pico Cell & 0.1 to 0.2 & 0.25 to 1 & 30 to 100 & Indoor/Outdoor & Cover a Building \\
Femtocell & 0.01 to 0.1 & 0.001 to 0.25 & 1 to 30 & Indoor & Cover a Flat \\
\hline
\end{tabular}

find users close by to transfer data. Today, Wi-Fi direct is used in such applications (e.g., Zapya [336]) for direct data transmission. Natural disasters and crisis management are other application types of D2D 5G networks that need to establish an infrastructure-less network when BSs are not available. In IoT, D2D can reduce delay, improve coverage, optimize energy efficiency and support QoS. Different applications like Vehicle to Vehicle (V2V) [337] can use a combination of D2D and Small Cell to improve the efficiency of the network infrastructure.

From a topology management perspective, combining D2D and Small Cell yields a network infrastructure which resembles with multi-gateway (i.e., multi-sink) WSNs. Similar to WSNs, this topology needs to be managed in order to satisfy different objectives, e.g., energy efficiency, handling bandwidth limitations, and delay reduction. These two technologies have the potential to be used for clustering in $5 \mathrm{G}$ networks.

To the best of our knowledge, the only paper that studies the effect of clustering in IoT 5G scenarios is [19]. The authors consider the use of clustering techniques in 5G IoT networks to support energy efficiency, distributed processing and hierarchical management of the network infrastructure. The authors studied five challenges including node heterogeneity, resource and financial cost of transmission, user utility, smart core network, and supporting mobility. However, Small Cell and D2D are not studied as a $5 \mathrm{G}$ infrastructure in the paper. In [338], the authors use a clustering method in D2D-assisted LTE-A networks for tie-breaking to maximize fairness and throughput. In their method, $\mathrm{CH}$ is opportunistically different at each scheduling frame to manage cellular traffic. In [339], the authors introduce user-centric cluster-based coordinated multi-points in 5G. Clusters can reduce the overhead of intercell coordination by limiting it to within the cluster only. In addition, they use the re-clustering method to distribute the traffic of cells to neighboring cells. Network densification is also a method that can use clustering techniques to improve the efficiency of spectrum utilization [340]. In [239], the authors use a clustering method to achieve green IoT in $5 \mathrm{G}$ networks and solve the problem of handover. In [341], Song et al. use a clustering method to minimize network energy cost and maximize connected IoT devices in 5G small cells based on the k-means algorithm. In [342], a clustering method is introduced based on supporting Massive MIMO in 5G. In [343], a method is introduced which uses cluster forms in $5 \mathrm{G}$ cellular networks to support $\mathrm{D} 2 \mathrm{D}$. The above literature show that clustering improve the efficiency of $5 \mathrm{G}$ by managing nodes and resources in each cluster locally. In this paper, we review the opportunities and challenges of clustering techniques in 5G networks based on Small Cell and D2D.

1) Importance of clustering in $5 G$ Networks: As mentioned above, in 5G settings that support Small Cell and D2D commu- 


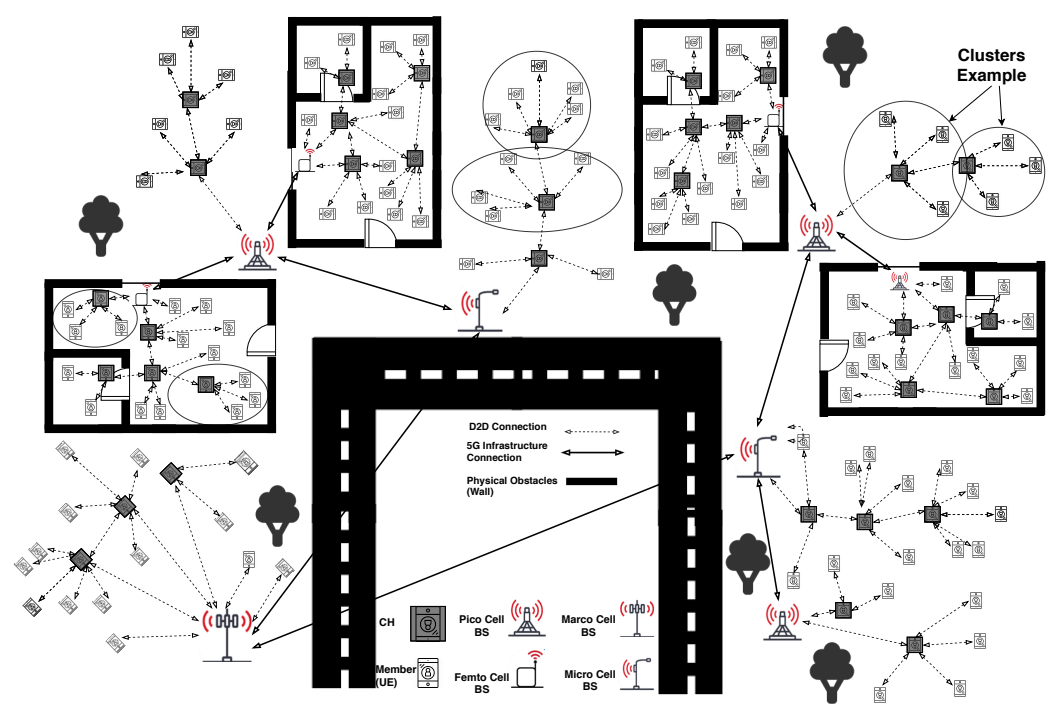

Fig. 6. An illustration of how IoT can be integrated with $5 \mathrm{G}$ based on clustering

nication technologies, clustering can be a foundation stone for topology management. Clustering techniques in 5G can support different applications and also improve the efficiency of communication based on grouping the nodes and hierarchical management. Below, we discuss the importance of clustering in $5 \mathrm{G}$ based on the different efficiency improvements achieved by $5 \mathrm{G}$ clustering.

- Applications: Not all applications over 5G need to use BS for data transmission, e.g., online group games. Based on 5G characteristics, D2D communication can be established among nodes that have Line of Sight (LoS). Clustering can connect different UEs to one UE as $\mathrm{CH}$ based on commonalities of applications or services.

- Improving massive MIMO efficiency: Massive MIMO use BSs with very large antenna arrays to communicate with several users in each time frequency [344]. With a large number of UEs which communicate with BS directly, interference can be quite high in BS, which can result in a smaller number of users being supported. Clustering can be considered as a solution to scale down the number of users connected directly, while the resulting multi-hop network topology can lead to increased delay in the network as discussed in [37]. Given the high speed of D2D communication in $5 \mathrm{G}$, clustering will not impose significant delay, in addition to the fact that the number of UEs connected directly to BS can be controlled, resulting in improving the efficiency of Massive MIMO.

- Caching: Leveraging small cells and D2D, it is possible to cache data in CHs. Cluster members can be UEs that need the same data. There are many types of applications that need caching, such as multimedia, online video conferencing, etc. Caching can help avoid data redundancy in data transmission. In hierarchical clustering techniques, caching can reduce the volume of data significantly by reusing data hop by hop. In addition, different services in the Fog can use cached data in CHs and avoid asking the Cloud to transfer. - Energy consumption: As one of the main challenges in $5 \mathrm{G}$, clustering can reduce overall network energy consumption through balancing energy consumption in nodes. Similarly, D2D and Small Cell are introduced to optimize energy consumption. Clustering techniques can be used in a $5 \mathrm{G}$ D2D network to delegate data transfer to the nodes that have higher energy resources.

- Spectrum efficiency: Spectrum efficiency is an important factor in $5 \mathrm{G}$ which is supported by the mmWave technology. By mmWave, UEs use a $10-300 \mathrm{GHz}$ band that supports a very fast gigabit-per-second data rate. In mmWave, communication between nodes is limited by their transmission power and physical obstacles. Using clustering techniques in such networks opens up for low range communication. This can support low multi-user interference (MUI) in ultradense networks like IoT. UEs can communicate with $\mathrm{CHs}$ by allocating different bands and adopting LOS or multihop D2D. In addition, in the case of having no LOS between nodes and $\mathrm{CH}$, intra-cluster routing can be provided by D2D communication.

- Resource usage efficiency: Each UE in 5G has specific resources that can be used in the network. Supporting D2D allows $5 \mathrm{G}$ to use the resources of UEs to support different services and applications. Clustering techniques can be used to group a set of nodes and assign a task to a node group.

- Network heterogeneity: In 5G, nodes support different network interfaces. Clustering techniques can be used to switch between network interfaces for data transmission. As an example, nodes can connect to $\mathrm{CH}$ using D2D and then use Wi-Fi direct to transfer data to the next hop.

Figure 6 shows an example of how clustering techniques can improve connectivity and efficiency of $5 \mathrm{G}$ networks.

2) Challenges of integrating clustering techniques with $5 G$ : Although using clustering techniques in 5G-IoT networks has many advantages as mentioned above, it also introduces a number of challenges:

- Heterogeneity: Most clustering techniques are designed for homogeneous networks, not considering requirements of 
other layers as a parameter in establishing clusters. As an example, mmWave technology can be confined by physical obstacles to connect to UEs. To establish clusters, physical obstacles can change the form of clusters, number of members, etc. which can cause inefficiency. On the other hand, clustering techniques should consider application services. As traditional clustering techniques are designed to establish clusters based on the network layer objectives like improving QoS, new parameters should be considered in cluster creation. As an example, to improve efficiency, clusters can be established to unite the nodes that use the same service as data generator or data consumer. These parameters make establishing and maintaining clusters more complicated and resource consuming.

- Interference: As 5G-IoT networks can possibly be a kind of Ultra-Dense networks, massive volumes of data in a cluster can cause interference among members. Generally, clusters can be established based on common applications among neighbor nodes, but also some applications can have a high volume of data to transfer which cause interference in an area. MUI reduction techniques might be considered in cluster establishment and member selection to distribute potential interference nodes among clusters. Using MUI as a parameter to establish clusters, may result in a clustering model in which each cluster contains members belonging to different applications which again would lead to interference reduction in a cluster. On the other hand, having nodes which consume or generate data of a specific application in a cluster can result in a higher chance of performing data aggregation, fusion, service migration efficiency, etc. Finding an optimal trade-off between reducing interference and establishing clusters based on common applications and services is a challenge that need to addressed.

- Scalability: with thousands of nodes in 5G-IoT networks, scalability is a great challenge. As 5G-IoT networks can be a UDN [345], the number of nodes and communication links per area are very high. As the radio range of the UEs should be limited to improve resource consumption and also reduce interference, the number of nodes per cluster and number of clusters per network would increase which cause more complexity of establishing clusters and connecting them to BSs. In addition, in $5 \mathrm{G}$ networks, there are several gateways in the network, basically BSs, which make the topology management more complicated. In massive-scale IoT applications, having multiple potential destinations, makes finding optimal routes to clusters and balancing the load of network efficiently a complex task.

- Dynamicity: Network dynamicity is a significant challenge in $5 \mathrm{G}$ networks due to the presence of mobile nodes, faulty nodes, etc. For example, some nodes are moved by humans as attached nodes like vehicles, cellphones, wearable sensors, etc. Routes, clusters and connections which transfer data through the mobile nodes need to be re-established after considerable network topology changes which cause high overhead and time complexity of re-clustering techniques. Having a dynamic network infrastructure in 5G IoT networks, new challenges emerge like channel impairment which should be considered in the design of a dynamic clustering technique.

- Hierarchical management: In UDN, network management is a challenge due to the scale of the network in terms of number of nodes and communication load. Hierarchical distributed management can address this challenge, applicable to cells of all sizes. Clusters should be established based on the different sizes of cells as the possible cost of causing cluster overlap. Reducing overlaps is a complicated process when establishing clusters within cells. Small Cell technology divides the network into cells and clustering divides the network into clusters. This implies that two identical node grouping techniques may simultaneously be used over a common network infrastructure. Applying the same clustering techniques simultaneously can cause unexpected efficiency problems. Overlaps and interference among clusters and cells may cause overheads and topology management problems. 5G networks supporting Small Cell technology are similar to multi-sink ad-hoc networks in which each BS is a sink. Therefore, selecting the best sink as the gateway of a packet stream would be challenging due to financial and resource costs.

\section{CONCLUSIONS}

Topology management is an important issue in the IoT networks that resemble ad-hoc networks, e.g., D2D-based IoT networks over cellular networks. Clustering is the most popular method for topology management, especially in WSNs. Some existing approaches for WSN clustering have the potential to be applied to IoT networks, even though special characteristics of IoT networks such as heterogeneity and nodes mobility make clustering a challenging issue in IoT. In this paper, we conducted a comprehensive survey on existing WSN clustering techniques and investigated their applicability to IoT networks. The survey work is conducted based on all the common clustering objectives (e.g., reducing energy consumption and load balancing), as well as the network properties impacting efficiency of IoT clustering (e.g., mobility). Classifying the well-known clustering literature shows that clustering can not only reduce energy consumption as its primary objective, but also achieve several other qualityrelated objectives. In addition, our investigations show that existing clustering techniques can contribute to better support of quality-related requirements of ad-hoc IoT networks, e.g., QoS and fault tolerance, although their high dynamicity and heterogeneity make clustering challenging. Moreover, we focused on the modern networking and computing paradigms integrated with IoT (i.e., Blockchain, SDN, NFV, Fog/Edge, and $5 \mathrm{G}$ ) and reviewed existing clustering-based approaches. Our thorough study shows that clustering can greatly contribute in better handling of design concerns related to scalability in these computing areas, from distributed control to service orchestration and resource allocation.

\section{ACKNOWLEDGMENT}

Research leading to these results has partially received funding from the EU ECSEL Joint Undertaking under grant agreement no 737459 (project Productive4.0) and from the Research Council of Norway 


\section{REFERENCES}

[1] Jie Lin et al. A survey on internet of things: Architecture, enabling technologies, security and privacy, and applications. IEEE Internet of Things Journal, 4(5):1125-1142, 2017.

[2] Mung Chiang and Tao Zhang. Fog and iot: An overview of research opportunities. IEEE Internet of Things Journal, 3(6):854-864, 2016.

[3] Tie Qiu et al. How can heterogeneous internet of things build our future: A survey. IEEE Communications Surveys \& Tutorials, 20(3):2011-2027, 2018.

[4] Raja Haseeb Javed et al. ApproxCT: Approximate Clustering Techniques for Energy Efficient Computer Vision in Cyber-Physical Systems. In 12th Int. Conf. on Open Source Systems and Technologies (ICOSST), pages 64-70. IEEE, 2018.

[5] Paolo Bellavista et al. Convergence of manet and wsn in iot urban scenarios. IEEE Sensors Journal, 13(10):3558-3567, 2013.

[6] Oladayo Bello et al. Intelligent device-to-device communication in the internet of things. IEEE Systems Journal, 10(3):1172-1182, 2014.

[7] Muhammad Mahtab Alam et al. A survey on the roles of communication technologies in iot-based personalized healthcare applications. IEEE Access, 6:36611-36631, 2018.

[8] Muhammad Khalil Afzal et al. Ieee access special section editorial: The new era of smart cities: Sensors, communication technologies, and applications. IEEE Access, 5:27836-27840, 2017.

[9] Armir Bujari et al. Would current ad-hoc routing protocols be adequate for the internet of vehicles? a comparative study. IEEE Internet of Things Journal, 5(5):3683-3691, 2018.

[10] Frank T Johnsen et al. Application of iot in military operations in a smart city. In 2018 International Conference on Military Communications and Information Systems (ICMCIS), pages 1-8. IEEE, 2018.

[11] Daniel G Reina et al. The role of ad hoc networks in the internet of things: A case scenario for smart environments. In Internet of things and inter-cooperative computational technologies for collective intelligence, pages 89-113. Springer, 2013.

[12] Wendi R. Heinzelman et al. Energy-efficient communication protocol for wireless microsensor networks. In System sciences, 2000. Proc. of the 33rd annual Hawaii Int. conference on, pages 1-10. IEEE, 2000.

[13] Ossama Younis and Sonia Fahmy. Heed: a hybrid, energy-efficient, distributed clustering approach for ad hoc sensor networks. IEEE Transactions on mobile computing, 3(4):366-379, 2004.

[14] A. Manjeshwar et al. Teen: Arouting protocol for enhanced efficiency in wireless sensor networks. In ipdps, pages 1-7. IEEE, 2001

[15] Ali Sh. Rostami et al. Survey on clustering in heterogeneous and homogeneous wireless sensor networks. The Journal of Supercomputing, 74(1):277323, 2018.

[16] M. M. Afsar et al. Clustering in sensor networks: A literature survey. Journal of Network and Computer Applications, 46:198-226, 2014.

[17] Xuxun Liu. A Survey on Clustering Routing Protocols in Wireless Sensor Networks. Sensors, 12(8):11113-11153, 2012.

[18] S. Arjunan et al. A survey on unequal clustering protocols in Wireless Sensor Networks. Journal of King Saud University - Computer and Information Sciences, 31(3):304-317, 2017.

[19] L. Xu. A Survey of Clustering Techniques in WSNs and Consideration of the Challenges of Applying Such to 5G IoT Scenarios. IEEE Internet of Things Journal, 4(5):1229-1249, 2017.

[20] M. Demirbas et al. FLOC: A Fast Local Clustering Service for Wireless Sensor Networks. First Int. Conf. on Broadband Networks, BroadNets 2004, pages 1-6, 2004.

[21] S. K. Singh et al. A survey on successors of LEACH protocol. IEEE Access, 5:4298-4328, 2017.

[22] Shio Kumar Singh et al. A survey of energy-efficient hierarchical cluster-based routing in wireless sensor networks. International Journal of Advanced Networking and Application (IJANA), 2(02):570-580, 2010.

[23] M. Younis et al. Topology management techniques for tolerating node failures in wireless sensor networks: A survey. Computer Networks, 58:254-283, 2014.

[24] S. M. AlMheiri et al. MANETs and VANETs clustering algorithms: A survey. In IEEE 8th GCC Conf. \& Exhibition, pages 1-6. IEEE, 2015.

[25] C. Cooper et al. A comparative survey of VANET clustering techniques. IEEE Communications Surveys \& Tutorials, 19(1):657 - 681, 2017.

[26] Dave Evans. The internet of things: How the next evolution of the internet is changing everything. CISCO white paper, 1(2011):1-11, 2011.
[27] Leila Shooshtarian et al. A clustering-based approach to efficient resource allocation in fog computing. In International Symposium on Pervasive Systems, Algorithms and Networks, pages 207-224. Springer, 2019.

[28] Ichraf El Haj Hamad et al. BTRMC, a bio-inspired trust and reputation model using clustering in WSNs. 2017 Int. Conf. on Smart, Monitored and Controlled Cities, SM2C 2017, 2017.

[29] Sameh Ben Fredj et al. A scalable IoT service search based on clustering and aggregation. In IEEE Int. Conf. on Green Computing and Communications and IEEE Internet of Things and IEEE Cyber, Physical and Social Computing, pages 403-410. IEEE, 2013.

[30] Neeraj Kumar et al. An intelligent clustering scheme for distributed intrusion detection in vehicular cloud computing. Cluster Computing, 18(3), 2015.

[31] Sen Zhou et al. Device clustering for fault monitoring in Internet of Things systems. IEEE World Forum on Internet of Things, WF-IoT 2015 - Proc., 2015.

[32] Lingjuan Lyu et al. Fog-empowered anomaly detection in IoT using hyperellipsoidal clustering. IEEE Internet of Things Journal, 4(5), 2017.

[33] Hanh Le et al. S-Web: An efficient and self-organizing wireless sensor network model. In Int. Conf. on Network-Based Information Systems, pages $179-188$. Springer, 2008.

[34] Z. Zhang et al. Energy-efficient multihop polling in clusters of two-layered heterogeneous sensor networks. IEEE Transactions on Computers, 57(2):231-245, 2008.

[35] D. Jia et al. Dynamic cluster head selection method for wireless sensor network. IEEE Sensors Journal, 16(8):2746-2754, 2016.

[36] M. Gavhale et al. Survey on algorithms for efficient cluster formation and cluster head selection in manet. Procedia Computer Science, 78:477-482, 2016

[37] A. Shahraki et al. A new approach for energy and delay trade-off intra-clustering routing in WSNs. Computers \& Mathematics with Applications, 62(4):1670-1676, 2011.

[38] Marjan Kuchaki Rafsanjani, Atieh Rezaei, Amin Shahraki, and Arsham Borumand Saeid. Qarima: A new approach to prediction in queue theory. Applied Mathematics and Computation, 244:514-525, 2014.

[39] E. Stuntebeck et al. Wireless underground sensor networks using commodity terrestrial motes. In Wireless Mesh Networks, 2006. WiMesh 2006. 2nd IEEE Workshop on, pages 112-114. IEEE, 2006.

[40] Peter J Bennett et al. Wireless sensor networks for underground railway applications: case studies in Prague and London. Smart Structures and Systems, 6(5-6):619-639, 2010.

[41] C. Uribe et al. Radio communication model for underwater WSN. In New Technologies, Mobility and Security, 3rd Int. Conf. on, pages 1-5. IEEE, 2009.

[42] A. Sharif et al. Wireless multimedia sensor network technology: A survey. In Industrial informatics, 7th IEEE Int. conference on, pages 606-613. IEEE, 2009.

[43] Feng Xia. QoS challenges and opportunities in wireless sensor/actuator networks. Sensors, 8(2):1099-1110, 2008.

[44] A. A. Abbasi et al. A survey on clustering algorithms for wireless sensor networks. Computer communications, 30(14-15):2826-2841, 2007.

[45] Taehyeun Park et al. Learning how to communicate in the internet of things: Finite resources and heterogeneity. IEEE Access, 4:7063-7073, 2016

[46] Amin Shahraki, Hamed Taherzadeh, and Øystein Haugen. Last significant trend change detection method for offline poisson distribution datasets. In 2017 International Symposium on Networks, Computers and Communications (ISNCC), pages 1-7. IEEE, 2017.

[47] Amin Shahraki et al. A comparative node evaluation model for highly heterogeneous massive-scale internet of things-mist networks. Transactions on Emerging Telecommunications Technologies, 2020.

[48] Amin Shahraki, Marius Geitle, and Øystein Haugen. A distributed fog node assessment model by using fuzzy rules learned by xgboost. In 2019 4th International Conference on Smart and Sustainable Technologies (SpliTech), pages 1-6. IEEE, 2019.

[49] Roberto Morabito et al. Evaluating performance of containerized iot services for clustered devices at the network edge. IEEE Internet of Things Journal, 4(4):1019-1030, 2017.

[50] Safwan M Ghaleb et al. Mobility management for iot: a survey. EURASIP Journal on Wireless Communications and Networking, 2016(1):1-25, 2016.

[51] Ricardo Silva et al. Mobility in wireless sensor networks-survey and proposal. Computer Communications, 52:1-20, 2014. 
[52] Kentaro Yanagihara et al. EACLE: Energy-aware clustering scheme with transmission power control for sensor networks. Wireless Personal Communications, 40(3):401-415, 2007.

[53] D. J. Baker et al. The Architectural Organization of a Mobile Radio Network via a Distributed Algorithm. IEEE Transactions on Communications, 29(11):1694-1701, 1981.

[54] C. Lin et al. Adaptive clustering for mobile wireless networks. IEEE Journal on Selected Areas in Communications, 15(7):1265-1275, 1997.

[55] R Nagpal et al. An algorithm for group formation in an amorphous computer. In 10th Int. Conf. on Parallel and Distributed Computing Systems, pages 1-4, 1998.

[56] S. Banerjee et al. A clustering scheme for hierarchical control in multi-hop wireless networks. Proc. IEEE INFOCOM 2001. Conf. on Computer Communications., 2:1028-1037, 2001.

[57] Beongku An and Symeon Papavassiliou. A mobility-based clustering approach to support mobility management and multicast routing in mobilead-hoc wireless networks. International Journal of Network Management, 11(6):387-395, 2001.

[58] S. Lindsey et al. Data gathering algorithms in sensor networks using energy metrics. IEEE Transactions on Parallel and Distributed Systems, 13(9):924-935, 2002.

[59] K. Xu. A heterogeneous routing protocol based on a new stable clustering scheme. In MILCOM 2002 Proc., volume 2, pages 838843. IEEE, 2002.

[60] A. Manjeshwar and D. P. Agrawal. APTEEN: A hybrid protocol for efficient routing and comprehensive information retrieval in wireless. In Proc. 16th Int. Parallel and Distributed Processing Symposium, pages 1-8. IEEE, 2002.

[61] H. Zhang et al. GS3: Scalable self-configuration and self-healing in wireless sensor networks. Computer Networks, 43(4):459-480, 2003.

[62] S Bandyopadhyay et al. Energy Efficient Hierarchical Clustering Algorithm for Wireless Sensor Networks. In Proc. IEEE INFOCOM, pages 1713-1723. IEEE, 2003.

[63] H. Chan et al. Ace: An emergent algorithm for highly uniform cluster formation. In European workshop on wireless sensor networks, pages 1-16. springer, 2004.

[64] G. Smaragdakis et al. SEP: A Stable Election Protocol for clustered heterogeneous wireless sensor networks . in proc. of the 2nd Int. workshop on SANPA, pages 1-11, 2004.

[65] P. Ding et al. Distributed energy-efficient hierarchical clustering for wireless sensor networks. In International conference on distributed computing in sensor systems, pages 322-339. Springer, 2005.

[66] Wang Ke et al. Attribute-based clustering for information dissemination in wireless sensor networks. In 2005 Sec. Аnпиаl IEEE Соттиnications Society Conf. on Sensor and AdHoc Communications and Networks, SECON 2005, volume 2005, pages 498-509. IEEE, 2005.

[67] S. Soro and W.B. Heinzelman. Prolonging the lifetime of wireless sensor networks via unequal clustering. In 19th IEEE Int. Parallel and Distributed Processing Symposium, pages 1-8. IEEE, 2005.

[68] H. Luo et al. Ttdd: Two-tier data dissemination in large-scale wireless sensor networks. Wireless networks, 11:161-175, 2005.

[69] S. D Muruganathan et al. A Centralized Energy-Effiecient Routing Protocol for Wireless Sensor Networks. IEEE Communications Magazine, 43(3):s8-s13, 2005.

[70] Mao Ye et al. An energy efficient clustering scheme in wireless sensor networks. Ad-Hoc and Sensor Wireless Networks, 3(2-3):99-119, 2007.

[71] Adel Youssef et al. Distributed formation of overlapping multi-hop clusters in wireless sensor networks. In IEEE GLOBECOM, pages 1-6. IEEE, 2006.

[72] B. Aoun et al. Clustering in WSN with latency and energy consumption constraints. Journal of Network and Systems Management, 14(3):415439, 2006.

[73] W. Choi et al. A distributed weighted clustering algorithm for mobile ad hoc networks. In Advanced Int'l Conf. on Telecommunications and Int'l Conf. on Internet and Web Applications and Services (AICT-ICIW'06), pages 1-6. IEEE, 2006.

[74] Sung Min Jung et al. The concentric clustering scheme for efficient energy consumption in the PEGASIS. In The 9th Int. Conf. on Advanced Communication Technology, pages 260-265. IEEE, 2007.

[75] M. Yu et al. A dynamic clustering and energy efficient routing technique for sensor networks. IEEE Transactions on Wireless Communications, 6(8):3069-3079, 2007.

[76] Y. Tian et al. Cluster-based information processing in wireless sensor networks: an energy-aware approach. Wireless Communications and Mobile Computing, 7(7):893-907, 2007.
[77] Tal Anker et al. Efficient clustering for improving network performance in wireless sensor networks. In European Conference on Wireless Sensor Networks, pages 221-236. Springer, 2008.

[78] Jiaxi You et al. Budget-based clustering with context-awareness for sensor networks. In Sixth Annual IEEE Int. Conf. on Pervasive Computing and Communications (PerCom), pages 306-311. IEEE, 2008

[79] Francesca Cuomo et al. Topology formation in ieee 802.15. 4: clustertree characterization. In Sixth Annual IEEE Int. Conf. on Pervasive Computing and Communications (PerCom), pages 276-281. IEEE, 2008.

[80] R. S. Marin-Perianu et al. Cluster-based service discovery for heterogeneous wireless sensor networks. International Journal of Parallel, Emergent and Distributed Systems, 23(4):325-346, 2008.

[81] Yan Jin et al. EEMC: An energy-efficient multi-level clustering algorithm for large-scale wireless sensor networks. Computer Networks, 52(3):542-562, 2008.

[82] Bencan Gong et al. Multihop routing protocol with unequal clustering for wireless sensor networks. In ISECS Int. Colloquium on Computing, Communication, Control, and Management, pages 552-556. IEEE, 2008 .

[83] Sungju Lee et al. An Energy-Efficient Distributed Unequal Clustering Protocol for Wireless Sensor Networks. Engineering and Technology, 2(12):2715-2719, 2008

[84] Sh. Varma et al. Base station initiated dynamic routing protocol for heterogeneous wireless sensor network using clustering. In Fourth Int Conf. on Wireless Communication and Sensor Networks, pages 1-6. IEEE, 2008

[85] Y. Wang et al. An Energy Efficient and Balance Hierarchical Unequal Clustering Algorithm for Large Scale Sensor Networks, 2009.

[86] G. Chen et al. An unequal cluster-based routing protocol in wireless sensor networks. Wireless Networks, 15:193-207, 2009.

[87] J. Lloret et al. A cluster-based architecture to structure the topology of parallel wireless sensor networks. Sensors, 9(12):10513-10544, 2009.

[88] M. Youssef et al. Overlapping multihop clustering for wireless senso networks. IEEE Transactions on Parallel and Distributed Systems, 20(12):1844-1856, 2009.

[89] Li-Qing Guo et al. Improvement on LEACH by combining adaptive cluster head election and two-hop transmission. In Int. Conf. on Machine Learning and Cybernetics (ICMLC), pages 1678-1683. IEEE, 2010.

[90] Samer A B Awwad et al. Cluster based routing protocol for mobile nodes in wireless sensor network. In Int. Symposium on Collaborative Technologies and Systems, pages 233-241. IEEE, 2009.

[91] D. Koutsonikolas et al. Hierarchical geographic multicast routing for wireless sensor networks. Wireless Networks, 16:449-466, 2010

[92] L. Buttyán et al. Position-Based Aggregator Node Election in Wireless Sensor Networks. International Journal of Distributed Sensor Networks, 6(1):1-15, 2010

[93] Ning $\mathrm{Xu}$ et al. Coverage and connectivity guaranteed topology control algorithm for cluster-based wireless sensor networks. Wireless Communications and Mobile Computing, 12(1):23-32, 2012

[94] H. Zhou et al. A novel stable selection and reliable transmission protocol for clustered heterogeneous wireless sensor networks. Computer Communications, 33(15):1843-1849, 2010.

[95] Y. Liu et al. Multi-layer clustering routing algorithm for wireless vehicular sensor networks. IET Communications, 4(7):810-816, 2010

[96] H. Bagci et al. An energy aware fuzzy unequal clustering algorithm for wireless sensor networks. In International Conference on Fuzzy Systems, pages 1-8. IEEE, 2010.

[97] Anshu Khare and Krishna M Sivalingam. On recovery of lost targets in a cluster-based wireless sensor network. In IEEE International Conference on Pervasive Computing and Communications Workshops (PERCOM Workshops), pages 208-213. IEEE, 2011.

[98] Yajie Ma et al. Distributed clustering-based aggregation algorithm for spatial correlated sensor networks. IEEE Sensors Journal, 11(3):641648, 2011.

[99] Sungryoul Lee et al. LUCA: An energy-efficient unequal clustering algorithm using location information for wireless sensor networks. Wireless Personal Communications, 56(4):715-731, 2011.

[100] S. Chaurasiya et al. An enhanced energy-efficient protocol with static clustering for WSN. In Int. Conf. on Information Networking ,, pages 58-63. IEEE, 2011.

[101] Jiguo Yu et al. An energy-aware distributed unequal clustering protocol for wireless sensor networks. International Journal of Distributed Sensor Networks, 7(1):1-8, 2011. 
[102] Jiguo Yu et al. An energy-driven unequal clustering protocol for heterogeneous wireless sensor networks. Journal of Control Theory and Applications, 9(1):133-139, 2011.

[103] W K Lai et al. Arranging cluster sizes and transmission ranges for wireless sensor networks. Information Sciences, 183(1):117-131, 2012.

[104] Ghalib A Shah et al. Cluster based coordination and routing framework for wireless sensor and actor networks. Wireless Communications and Mobile Computing, 11(8):1140-1154, 2011.

[105] S. Deng et al. Mobility-based clustering protocol for wireless sensor networks with mobile nodes. IET Wireless Sensor Systems, 1(1):39 47, 2011.

[106] T. Liu et al. An energy-balancing clustering approach for gradientbased routing in wireless sensor networks. Computer Communications, 35(17):2150-2161, 2012

[107] A. Wang et al. A clustering algorithm based on energy information and cluster heads expectation for wireless sensor networks. Computers \& Electrical Engineering, 38(3):662-671, 2012.

[108] Hoda Taheri et al. An energy-aware distributed clustering protocol in wireless sensor networks using fuzzy logic. Ad Hoc Networks, 10(7):1469-1481, 2012.

[109] J. S. Lee et al. Fuzzy-logic-based clustering approach for wireless sensor networks using energy predication. IEEE Sensors Journal, 12(9):2891 - 2897, 2012.

[110] Zhu Yong and Qing Pei. A energy-efficient clustering routing algorithm based on distance and residual energy for Wireless Sensor Networks. Procedia Engineering, 29:1882-1888, 2012.

[111] A. U. R Khan et al. Clustering-based power-controlled routing for mobile wireless sensor networks. International journal of communication systems, 25(4):529-542, 2012.

[112] J. Xu et al. Improvement of leach protocol for wsn. In 9th Int. Conf. on Fuzzy Systems and Knowledge Discovery, pages 2174-2177. IEEE, 2012.

[113] Song Mao et al. An improved fuzzy unequal clustering algorithm for wireless sensor network. Mobile Networks and Applications, 18(2):206214, 2013

[114] Huan Li et al. COCA: Constructing optimal clustering architecture to maximize sensor network lifetime. Computer Communications, 36(3):256-268, 2013.

[115] Y. Chen et al. Distributed Clustering With Directional Antennas for Wireless Sensor Networks. IEEE Sensors Journal, 13(6):2166-2180, 2013.

[116] S. Sheik Faritha Begum et al. Modified load-balanced clustering algorithm with distributed self-organization for wireless sensor networks. International Journal of Pharmacy and Technology, 8(4):23349-23356, 2016.

[117] S. S. Wang et al. LCM: A link-aware clustering mechanism for energyefficient routing in wireless sensor networks. IEEE Sensors Journal, 13(2):728 - 736, 2013.

[118] D. Mahmood et al. MODLEACH: A variant of LEACH for WSNs. In 8th Int. Conf. on Broadband, Wireless Computing, Communication and Applications, BWCCA, pages 158-163. IEEE, 2013.

[119] B. Manzoor et al. Q-LEACH: A new routing protocol for WSNs Procedia Computer Science, 19:926-931, 2013.

[120] Nadeem Javaid et al. EDDEEC: Enhanced developed distributed energy-efficient clustering for heterogeneous wireless sensor networks. Procedia Computer Science, 19:914-919, 2013.

[121] M. A. Jan et al. PASCCC: Priority-based application-specific congestion control clustering protocol. Computer Networks, 74:92-102, 2014.

[122] Suraj Sharma and Sanjay Kumar Jena. Cluster based multipath routing protocol for wireless sensor networks. ACM SIGCOMM Computer Communication Review, 45(2):14-20, 2015.

[123] P. Kuila et al. A novel differential evolution based clustering algorithm for wireless sensor networks. Applied Soft Computing, 25:414-425, 2014.

[124] Sachin Gajjar et al. FAMACRO: Fuzzy and ant colony optimization based MAC/routing cross-layer protocol for wireless sensor networks. Procedia Computer Science, 46:1014-1021, 2015.

[125] J RejinaParvin et al. Particle swarm optimization-based clustering by preventing residual nodes in wireless sensor networks. IEEE sensors journal, 15(8):4264-4274, 2015.

[126] Davood Izadi et al. An alternative clustering scheme in WSN. IEEE sensors journal, 15(7):4148-4155, 2015.

[127] Chuan Zhu et al. A Tree-Cluster-Based Data-Gathering Algorithm for Industrial WSNs With a Mobile Sink. IEEE Access, 3(1):381-396, 2015 .
[128] Md Azharuddin et al. Energy efficient fault tolerant clustering and routing algorithms for wireless sensor networks. Computers and Electrical Engineering, 41:177-190, 2015.

[129] De gan Zhang et al. A new clustering routing method based on PECE for WSN. Eurasip Journal on Wireless Communications and Networking, 2015(1):1-13, 2015.

[130] R. R. Sahoo et al. A bio inspired and trust based approach for clustering in WSN. Natural Computing, 15:423-434, 2016.

[131] Dan Liu et al. Cluster-based energy-efficient transmission using a new hybrid compressed sensing in wsn. In IEEE conference on computer communications workshops (INFOCOM WKSHPS), pages 372-376. IEEE, 2016.

[132] Yanqiu Huang et al. Pkf-st: A communication cost reduction scheme using spatial and temporal correlation for wireless sensor networks. In EWSN, pages 47-52, 2016.

[133] Vrinda Gupta and Rajoo Pandey. An improved energy aware distributed unequal clustering protocol for heterogeneous wireless sensor networks. Engineering Science and Technology, an International Journal, 19(2):1050-1058, 2016.

[134] R. Logambigai et al. Fuzzy logic based unequal clustering for wireless sensor networks. Wireless Networks, 22(3):945957, 2016.

[135] B. Baranidharan et al. DUCF: Distributed load balancing Unequal Clustering in wireless sensor networks using Fuzzy approach. Applied Soft Computing Journal, 40:495-506, 2016.

[136] Srikanth Jannu and Prasanta K Jana. A grid based clustering and routing algorithm for solving hot spot problem in wireless sensor networks. Wireless Networks, 22(6):1901-1916, 2016.

[137] P. C. Srinivasa Rao and Haider Banka. Novel chemical reaction optimization based unequal clustering and routing algorithms for wireless sensor networks. Wireless Networks, 23(3):759778, 2017.

[138] Nabil Sabor et al. An Unequal Multi-hop Balanced Immune Clustering protocol for wireless sensor networks. Applied Soft Computing Journal, 43:372-389, 2016

[139] A. Guiloufi et al. An Energy-Efficient Unequal Clustering Algorithm Using Śierpinski Trianglefor WSNs. Wireless Personal Communications, 88:449465, 2016.

[140] Hui Xia et al. Energy-Efficient Routing Algorithm Based on Unequal Clustering and Connected Graph in Wireless Sensor Networks. International Journal of Wireless Information Networks, 23(2):141150, 2016.

[141] D S Mantri et al. Mobility and heterogeneity aware cluster-based data aggregation for wireless sensor network. Wireless Personal Communications, 86:975-993, 2016.

[142] P. Nayak et al. A Fuzzy Logic-Based Clustering Algorithm for WSN to Extend the Network Lifetime. IEEE Sensors Journal, 16(1):137 144, 2016.

[143] R. Zhang et al. NDCMC: A Hybrid Data Collection Approach for Large-Scale WSNs Using Mobile Element and Hierarchical Clustering. IEEE Internet of Things Journal, 3(4):533-543, 2016.

[144] Nabajyoti Mazumdar and Hari Om. Distributed fuzzy logic based energy-aware and coverage preserving unequal clustering algorithm for wireless sensor networks. International Journal of Communication Systems, 30(13):e3283, 2017.

[145] PC S. Rao et al. A particle swarm optimization based energy efficient cluster head selection algorithm for wireless sensor networks. Wireless networks, 23:2005-2020, 2017.

[146] D. Lin et al. A game theory based energy efficient clustering routing protocol for WSNs. Wireless Networks, 23:1101-1111, 2017.

[147] M. Bala Krishna and M. N. Doja. Deterministic K-means secure coverage clustering with periodic authentication for wireless sensor networks. International Journal of Communication Systems, 30(4):116,2017

[148] A. Shahraki et al. Hierarchical distributed management clustering protocol for wireless sensor networks. Telecommunication Systems, 65:193-214, 2017.

[149] Xiaohui Yuan et al. A Genetic Algorithm-Based, Dynamic Clustering Method Towards Improved WSN Longevity. Journal of Network and Systems Management, 25(1):21-46, 2017

[150] M. Shokouhifar et al. Optimized sugeno fuzzy clustering algorithm for wireless sensor networks. Engineering Applications of Artificial Intelligence, 60:16-25, 2017.

[151] Jin Wang et al. Particle swarm optimization based clustering algorithm with mobile sink for WSNs. Future Generation Computer Systems, 76:452-457, 2017. 
[152] K. A Darabkh et al. EA-CRP: A novel energy-aware clustering and routing protocol in wireless sensor networks. Computers \& Electrical Engineering, 72:702-718, 2017.

[153] PC Srinivasa Rao and Haider Banka. Energy efficient clustering algorithms for wireless sensor networks: novel chemical reaction optimization approach. Wireless Networks, 23(2):433-452, 2017.

[154] Jin Wang et al. Energy-efficient cluster-based dynamic routes adjustment approach for wireless sensor networks with mobile sinks. The Journal of Supercomputing, 73(7):32773290, 2017.

[155] A. Abuarqoub et al. Dynamic clustering and management of mobile wireless sensor networks. Computer Networks, 117:62-75, 2017.

[156] S. Singh et al. HSCA: a novel harmony search based efficient clustering in heterogeneous WSNs. Telecommunication Systems, 67:651667, 2018.

[157] D. Agrawal. FUCA: Fuzzy-based unequal clustering algorithm to prolong the lifetime of wireless sensor networks. International Journal of Communication Systems, 31(2):e3448, 2018.

[158] Melika Khabiri and Ali Ghaffari. Energy-Aware Clustering-Based Routing in Wireless Sensor Networks Using Cuckoo Optimization Algorithm. Wireless Personal Communications, 98(3):2473-2495, 2018.

[159] John Anzola et al. A clustering WSN routing protocol based on k-d tree algorithm. Sensors, 18(9):1-26, 2018.

[160] T. Gaber et al. Trust-based secure clustering in WSN-based intelligent transportation systems. Computer Networks, 146:151-158, 2018.

[161] K. Muthukumaran et al. An energy efficient clustering scheme using multilevel routing for wireless sensor network. Computers and Electrical Engineering, 69:642-652, 2018.

[162] Peyman Neamatollahi et al. Distributed Clustering-Task Scheduling for Wireless Sensor Networks Using Dynamic Hyper Round Policy. IEEE Transactions on Mobile Computing, 17(2):334-347, 2018.

[163] Niayesh Gharaei et al. Collaborative Mobile Sink Sojourn Time Optimization Scheme for Cluster-Based Wireless Sensor Networks. IEEE Sensors Journal, 18(16):6669 - 6676, 2018.

[164] S. K. Singh et al. An Energy Efficient Protocol to Mitigate Hot Spot Problem Using Unequal Clustering in WSN. Wireless Personal Communications, 101:799-827, 2018.

[165] S K Preeth et al. An adaptive fuzzy rule based energy efficient clustering and immune-inspired routing protocol for WSN-assisted IoT system. Journal of Ambient Intelligence and Humanized Computing, pages 1-13, 2018.

[166] J. Lin et al. Efficient fault-tolerant routing in iot wireless sensor networks based on bipartite-flow graph modeling. IEEE Access, 7:14022-14034, 2019.

[167] Quan Wang et al. An energy-efficient compressive sensing-based clustering routing protocol for wsns. IEEE Sensors Journal, 19(10):39503960, 2019.

[168] Kh. A. Darabkh et al. BPA-CRP: A balanced power-aware clustering and routing protocol for wireless sensor networks. Ad Hoc Networks, 82:155-171, 2019

[169] D. Lin. An Energy-Efficient Clustering Algorithm Combined Game Theory and Dual-Cluster-Head Mechanism for WSNs. IEEE Access, 7:49894 - 49905, 2019.

[170] S. Randhawa et al. MLBC: Multi-objective Load Balancing Clustering technique in Wireless Sensor Networks. Applied Soft Computing, 74:66-89, 2019.

[171] Rashmi Sharan Sinha et al. A survey on lpwa technology: Lora and nb-iot. Ict Express, 3(1):14-21, 2017

[172] Sh. Manurkar. Building IoT nodes-a flexible approach. In Proc. of the 17th ACM/IEEE Int. Conf. on Information Processing in Sensor Networks, pages 154-155, 2018.

[173] Ilhem Souissi et al. A multi-level study of information trust models in wsn-assisted iot. Computer Networks, 151:12-30, 2019.

[174] Güngör Yıldırım and Yetkin Tatar. On wsn heterogeneity in iot and cpss. In Int. Conf. on Computer Science and Engineering (UBMK), pages 1020-1024. IEEE, 2017.

[175] R. Tandon et al. Weight based clustering in wireless sensor networks. In National Conf. on Communications, pages 1-5. IEEE, 2013.

[176] Amir Taherkordi, Frank Eliassen, and Geir Horn. From iot big data to iot big services. In Proceedings of the Symposium on Applied Computing, SAC '17, page 485491, 2017.

[177] Hitesh Mohapatra and Amiya Kumar Rath. Fault Tolerance Through Energy Balanced Cluster Formation (EBCF) in WSN. In Smart Innovations in Communication and Computational Sciences, pages 313-321. Springer, 2019.
[178] Fang Liu and Tong Li. A clustering k-anonymity privacy-preserving method for wearable iot devices. Security and Communication Networks, 2018:1-8, 2018.

[179] S M Ghaleb et al. Mobility management for IoT: a survey. EURASIP Journal on Wireless Communications and Networking, 2016(1):1-25, 2016.

[180] Abida Sharif et al. Internet of things enabled vehicular and ad hoc networks for smart city traffic monitoring and controlling: a review. International Journal of Advanced Networking and Applications, 10(3):3833-3842, 2018

[181] Abhijit Sarma et al. Deciding handover points based on context-aware load balancing in a wifi-wimax heterogeneous network environment IEEE Transactions on Vehicular Technology, 65(1):348-357, 2015.

[182] Stefan Feirer and Thilo Sauter. Seamless handover in industrial wlan using ieee $802.11 \mathrm{k}$. In IEEE 26th international symposium on industrial electronics (ISIE), pages 1234-1239. IEEE, 2017.

[183] Afonso Oliveira and Teresa Vazão. Low-power and lossy networks under mobility: A survey. Computer networks, 107:339-352, 2016.

[184] Ved P Kafle et al. Internet of things standardization in itu and prospective networking technologies. IEEE Communications Magazine, 54(9):43-49, 2016.

[185] S. Kumar et al. Using clustering approaches for response time aware job scheduling model for internet of things (IoT). International Journal of Information Technology, 9:177-195, 2017.

[186] Vijay Laxmi Kalyani et al. Iot: machine to machine (m2m), device to device (d2d) internet of everything (ioe) and human to human (h2h): future of communication. Journal of Management Engineering and Information Technology, 2(6):17-23, 2015.

[187] J. W. Hui et al. IPv6 in low-power wireless networks. Proc. of the IEEE, 98(11):1865 - 1878, 2010.

[188] Felix Jonathan Oppermann et al. A decade of wireless sensing applications: Survey and taxonomy. In The Art of Wireless Sensor Networks, pages 11-50. Springer, 2014

[189] X. Sun et al. EdgeIoT: Mobile edge computing for the Internet of Things. IEEE Communications Magazine, 54(12):22 - 29, 2016.

[190] Bhaskar P Deosarkar et al. Clusterhead selection in clustering algorithms for wireless sensor networks: A survey. In Int. Conf. on Computing, Communication and Networking, pages 1-8. IEEE, 2008.

[191] Qingjian Ni et al. A novel cluster head selection algorithm based on fuzzy clustering and particle swarm optimization. IEEE/ACM Transactions on Computational Biology and Bioinformatics, 14(1):7684, 2015.

[192] K. Jung et al. Improving adaptive cluster head selection of teen protocol using fuzzy logic for WMSN. Multimedia Tools and Applications, $76: 1817518190,2017$

[193] S. Sharma et al. Artificial neural network based cluster head selection in wireless sensor network. International Journal of Computer Applications, 119(4):34-41, 2015.

[194] K Vijayalakshmi et al. A multi objective Tabu particle swarm optimization for effective cluster head selection in WSN. Cluster Computing, 22:1227512282, 2018.

[195] T Shankar et al. Hybrid HSA and PSO algorithm for energy efficient cluster head selection in wireless sensor networks. Swarm and Evolutionary Computation, 30:1-10, 2016.

[196] Yifeng Zhao et al. Distributed dynamic cluster-head selection and clustering for massive iot access in $5 \mathrm{~g}$ networks. Applied Sciences, 9(1):1-15, 2019

[197] Qian Li et al. EnergIoT: A solution to improve network lifetime of IoT devices. Pervasive and Mobile Computing, 42:124-133, 2017.

[198] Jeong-Won Kim. A cluster head replacement based on threshold in the internet of things. The Journal of the Korea institute of electronic communication sciences, 9(11):1241-1248, 2014.

[199] Jine Tang et al. An energy efficient hierarchical clustering index tree for facilitating time-correlated region queries in the Internet of Things. Journal of Network and Computer Applications, 40(1):1-11, 2014.

[200] Jing Zhang, Xin Feng, et al. A grid-based clustering algorithm via load analysis for industrial internet of things. IEEE Access, 6:13117-13128, 2018.

[201] V. Loscrì et al. A two-levels hierarchy for low-energy adaptive clustering hierarchy (TL-LEACH). In IEEE 62nd Vehicular Technology Conf., pages 1809-1813. IEEE, 2005.

[202] A. Bagula et al. Service-aware clustering: An energy-efficient model for the internet-of-things. Sensors, 16:1-24, 2015.

[203] Nandhakumar Ramachandran et al. Sensor search using clustering technique in a massive iot environment. In Industry Interactive Innovations in Science, Engineering and Technology, pages 271-281. Springer, 2018. 
[204] Tao Han et al. A novel cluster head selection technique for edgecomputing based iomt systems. Computer Networks, 158:114-122, 2019.

[205] Arash Bozorgchenani et al. An energy-aware offloading clustering approach (eaoca) in fog computing. In Int. Symposium on Wireless Communication Systems (ISWCS), pages 390-395. IEEE, 2017.

[206] Omar Said et al. Iot-rtp and iot-rtcp: Adaptive protocols for multimedia transmission over internet of things environments. IEEE access, 5:16757-16773, 2017.

[207] Hyung-Sin Kim et al. Challenging the ipv6 routing protocol for lowpower and lossy networks (rpl): A survey. IEEE Communications Surveys \& Tutorials, 19(4):2502-2525, 2017.

[208] Harith Kharrufa et al. Rpl-based routing protocols in iot applications: A review. IEEE Sensors Journal, 19(15):5952-5967, 2019.

[209] Oana Iova et al. Rpl: The routing standard for the internet of things... or is it? IEEE Communications Magazine, 54(12):16-22, 2016.

[210] S. Jannu et al. A grid based clustering and routing algorithm for solving hot spot problem in wireless sensor networks. Wireless Networks, 22(6):1901-1916, 2016

[211] A Sebastian and S Sivagurunathan. A survey on load balancing schemes in rpl based internet of things. International Journal of Scientific Research in Network Security and Communication, 6(3):4349, 2018.

[212] J. Li et al. A clustering based routing algorithm in IoT aware Wireless Mesh Networks. Sustainable Cities and Society, 40:657-666, February 2018

[213] Behrouz Pourghebleh and Vahideh Hayyolalam. A comprehensive and systematic review of the load balancing mechanisms in the internet of things. Cluster Computing, 23:641661, 2019.

[214] Marjan Farahani and Akbar Ghaffarpour Rahbar. Double leveled unequal clustering with considering energy efficiency and load balancing in dense iot networks. Wireless Personal Communications, 106(3):1183-1207, 2019.

[215] Chinyang Henry Tseng. Multipath load balancing routing for internet of things. Journal of Sensors, 2016:1-8, 2016.

[216] S Santiago et al. Ealba: energy aware load balancing algorithm for iot networks. In Proc. of the 2018 Int. Conf. on Mechatronic Systems and Robots, pages 46-50, 2018.

[217] Thair A Al-Janabi et al. Optimised clustering algorithm-based centralised architecture for load balancing in iot network. In Int. Symposium on Wireless Communication Systems (ISWCS), pages 269-274. IEEE, 2017.

[218] Ahmed Aziz et al. Effective algorithm for optimizing compressive sensing in IoT and periodic monitoring applications. Journal of Network and Computer Applications, 126:12-28, 2019.

[219] Hamid Tahaei et al. The rise of traffic classification in iot networks: A survey. Journal of Network and Computer Applications, 154:102538, 2020.

[220] Fatima Hussain et al. A new block-based reinforcement learning approach for distributed resource allocation in clustered iot networks. IEEE Transactions on Vehicular Technology, 69(3):2891 - 2904, 2020.

[221] B. H Al-Qarni et al. An efficient networking protocol for internet of things to handle multimedia big data. Multimedia Tools and Applications, 78:30039-30056, 2018.

[222] Shesha Sreenivasamurthy and Katia Obraczka. Clustering for load balancing and energy efficiency in iot applications. In IEEE 26th Int. Symposium on Modeling, Analysis, and Simulation of Computer and Telecommunication Systems (MASCOTS), pages 319-332. IEEE, 2018.

[223] S. Halder et al. LiMCA: an optimal clustering algorithm for lifetime maximization of internet of things. Wireless Networks, 25:4459-4477, 2018

[224] Feyza Yildirim Okay and Suat Ozdemir. Routing in fog-enabled iot platforms: A survey and an sdn-based solution. IEEE Internet of Things Journal, 5(6):4871-4889, 2018.

[225] Chi-Sheng Shih et al. Data alignment for multiple temporal data streams without synchronized clocks on iot fusion gateway. In IEEE Int. Conf. on Data Science and Data Intensive Systems, pages 667-674. IEEE, 2015.

[226] F. Jalali et al. Cognitive iot gateways: automatic task sharing and switching between cloud and edge/fog computing. In Proceedings of the SIGCOMM Posters and Demos, pages 121-123. ACM, 2017.

[227] K Sneha and BG Prasad. Load balanced efficient routing technique for mobility based rpl. In Int. Conf. on Smart Trends for Information Technology and Computer Communications, pages 225-235. Springer, 2017
[228] Mohammed Zaki Hasan and Fadi Al-Turjman. Optimizing multipath routing with guaranteed fault tolerance in internet of things. IEEE Sensors Journal, 17(19):6463-6473, 2017.

[229] Thaha Muhammed et al. Hcdsr: A hierarchical clustered fault tolerant routing technique for iot-based smart societies. In Smart Infrastructure and Applications, pages 609-628. Springer, 2020.

[230] S. Chouikhi et al. A survey on fault tolerance in small and large scale wireless sensor networks. Computer Communications, 69:22-37, 2015.

[231] J. Chudzikiewicz et al. Fault-tolerant techniques for the Internet of Military Things. In 2nd World Forum on Internet of Things (WF-IoT), pages 496-501. IEEE, 2015.

[232] Rajat Subhra Chakraborty et al. Security and fault tolerance in internet of things. Springer, 2019.

[233] Hala Hamadeh et al. Area, energy, and time assessment for a distributed tpm for distributed trust in iot clusters. Integration, 58:267-273, 2017.

[234] Vitor Barbosa Souza et al. Proactive vs reactive failure recovery assessment in combined Fog-to-Cloud (F2C) systems. In 22nd Int. Workshop on Computer Aided Modeling and Design of Communication Links and Networks (CAMAD), pages 1-5. IEEE, 2017.

[235] Ling Li, Shancang Li, and Shanshan Zhao. QoS-aware scheduling of services-oriented internet of things. IEEE Transactions on Industrial Informatics, 10(2):1497 - 1505, 2014.

[236] Roberto Morabito et al. Evaluating performance of containerized IoT services for clustered devices at the network edge. IEEE Internet of Things Journal, 4(4):1019-1030, 2017.

[237] T. López et al. Resource management in the internet of things: Clustering, synchronisation and software agents. In Architecting the Internet of Things, pages 159-193. Springer, 2011.

[238] A. Shahraki et al. Social ethics in Internet of Things: An outline and review. In Industrial Cyber-Physical Systems (ICPS), pages 509-516. IEEE, 2018.

[239] S. Din et al. MGR: Multi-parameter Green Reliable communication for Internet of Things in 5G network. Journal of Parallel and Distributed Computing, 118:34-45, 2018.

[240] Mauro Conti et al. Remi: a reliable and secure multicast routing protocol for iot networks. In Proceedings of the 12th Int. Conf. on Availability, Reliability and Security, pages 1-8, 2017.

[241] Liumeng Song et al. Qos-aware energy-efficient cooperative scheme for cluster-based iot systems. IEEE Systems Journal, 11(3):1447-1455, 2017.

[242] Nandhakumar Ramachandran et al. Delay-aware heterogeneous clusterbased data acquisition in internet of things. Computers \& Electrical Engineering, 65:44-58, 2018.

[243] Xin Feng et al. An Unequal Clustering Algorithm Concerned With Time-Delay for Internet of Things. IEEE Access, 6:33895 - 33909, 2018.

[244] Faouzi Hidoussi et al. PEAL: Power Efficient and Adaptive Latency Hierarchical Routing Protocol for Cluster-Based WSN. Wireless Personal Communications, 96(4):49294945, 2017.

[245] Ricardo Severino et al. Dynamic cluster scheduling for cluster-tree WSNs. SpringerPlus, 3(1):1-17, 2014.

[246] M. Aslam et al. CEEC: Centralized energy efficient clustering a new routing protocol for WSNs. In 9th Annual IEEE Communications Society Conf on Sensor, Mesh and Ad Hoc Communications and Networks (SECON), pages 103-105. IEEE, 2012.

[247] Syed Bilal Shah et al. Energy and interoperable aware routing for throughput optimization in clustered iot-wireless sensor networks. Future Generation Computer Systems, 81:372-381, 2018.

[248] Rowayda A Sadek. Hybrid energy aware clustered protocol for iot heterogeneous network. Future Computing and Informatics Journal, 3(2):166-177, 2018

[249] Dnyaneshwar Mantri et al. MHBCDA: Mobility and heterogeneity aware bandwidth efficient cluster based data aggregation for wireless sensor network. In Wireless VITAE, pages 1-5. IEEE, 2013.

[250] Muwonge S Bernard et al. Qos strategies for wireless multimedia sensor networks in the context of iot. In Int. Conf. on e-Infrastructure and e-Services for Developing Countries, pages 228-253. Springer, 2018.

[251] Zeyu Sun et al. An optimized clustering communication protocol based on intelligent computing in information-centric internet of things. IEEE Access, 7:28238-28249, 2019.

[252] Krzysztof Nowicki and Tadeus Uhl. Qos/qoe in the heterogeneous internet of things (iot). In Beyond the Internet of Things, pages 165196. Springer, 2017.

[253] Pongnapat Jutadhamakorn et al. A scalable and low-cost mqtt broker clustering system. In 2nd Int. Conf. on Information Technology, pages 1-5. IEEE, 2017. 
[254] Sameh Ben Fredj et al. A scalable iot service search based on clustering and aggregation. In IEEE Int. Conf. on Green Computing and Communications and IEEE Internet of Things and IEEE Cyber, Physical and Social Computing, pages 403-410. IEEE, 2013.

[255] J Sathish Kumar et al. Hierarchical clustering for dynamic and heterogeneous internet of things. Procedia Computer Science, 93:276282, 2016.

[256] Mohammad Mozaffari et al. Mobile internet of things: Can uavs provide an energy-efficient mobile architecture? In IEEE global communications conference (GLOBECOM), pages 1-6. IEEE, 2016.

[257] Jiachen Yang et al. Optimization of real-time traffic network assignment based on iot data using dbn and clustering model in smart city. Future Generation Computer Systems, 108:976-986, 2017.

[258] N. Jabeur et al. Toward a bio-inspired adaptive spatial clustering approach for IoT applications. Future Generation Computer Systems, 107:736-744, 2016.

[259] Amir Taherkordi, Reza Mohammadi, and Frank Eliassen. A communication-efficient distributed clustering algorithm for sensor networks. AINAW '08, page 634638, 2008.

[260] Haejoon Jung and In-Ho Lee. Performance analysis of threedimensional clustered device-to-device networks for internet of things. Wireless Communications and Mobile Computing, 2017:1-10, 2017.

[261] Muhammad Junaid Farooq et al. A multi-layer feedback system approach to resilient connectivity of remotely deployed mobile internet of things. IEEE Transactions on Cognitive Communications and Networking, 4(2):422-432, 2018.

[262] Mona Jaber et al. 5g backhaul challenges and emerging research directions: A survey. IEEE access, 4:1743 - 1766, 2016

[263] J. Zhang et al. A Grid-Based Clustering Algorithm via Load Analysis for Industrial Internet of Things. IEEE Access, 6:13117-13128, 2018.

[264] Chittabrata Ghosh et al. Method and apparatus for iot device clustering, June 29 2017. US Patent App. 14/757,628.

[265] Al-Sakib Khan Pathan. Security of self-organizing networks: MANET, WSN, WMN, VANET. CRC press, 2016.

[266] Paulo Gaona-García et al. Analysis of security mechanisms based on clusters iot environments. International Journal of Interactive Multimedia \& Artificial Intelligence, 4(3):55-60, 2017.

[267] T. Gaber et al. Trust-based secure clustering in WSN-based intelligent transportation systems. Computer Networks, 146:151-158, 2018.

[268] N. Abhishek et al. An intrusion detection system for detecting compromised gateways in clustered iot networks. In 2018 IEEE International Workshop Technical Committee on Communications Quality and Reliability (CQR), pages 1-6. IEEE, 2018.

[269] Chang-Sik Choi et al. Densification leveraging mobility: An iot architecture based on mesh networking and vehicles. In Proceedings of the Eighteenth ACM International Symposium on Mobile Ad Hoc Networking and Computing, pages 71-80, 2018.

[270] D. Mantri et al. Two tier cluster based data aggregation (TTCDA) for efficient bandwidth utilization in wireless sensor network. Wireless Personal Communications, 75:2589-2606, 2014.

[271] Liumeng Song et al. Energy efficiency cooperative scheme for clusterbased capillary networks in internet of things systems. In IEEE 27th Annual Int. Symposium on Personal, Indoor, and Mobile Radio Communications (PIMRC), pages 1-6. IEEE, 2016.

[272] Junyu Liu et al. Network densification in 5g: From the shortrange communications perspective. IEEE Communications Magazine, 55(12):96-102, 2017.

[273] Ghulam Shabbir et al. Network performance enhancement of multisink enabled low power lossy networks in sdn based internet of things. International Journal of Parallel Programming, 48:367-398, 2018.

[274] Tie Qiu et al. A novel shortcut addition algorithm with particle swarm for multisink internet of things. IEEE Transactions on Industrial Informatics, 16(5):3566 - 3577, 2019.

[275] Yoonyoung Sung et al. A multi-hop clustering mechanism for scalable iot networks. Sensors, 18(4):1-15, 2018.

[276] Nor Aida Mahiddin et al. An internet access solution: Manet routing and a gateway selection approach for disaster scenarios. The Review of Socionetwork Strategies, 11(1):47-64, 2017.

[277] D. Zhaoyang et al. Blockchain: a secure, decentralized, trusted cyber infrastructure solution for future energy systems. Journal of Modern Power Systems and Clean Energy, 6(5):958 - 967, 2018

[278] Amin Shahraki, Mahmoud Abbasi, and Øystein Haugen. Boosting algorithms for network intrusion detection: A comparative evaluation of real adaboost, gentle adaboost and modest adaboost. Engineering Applications of Artificial Intelligence, 94:103770, 2020.
[279] M. Hammi et al. BCTrust: A decentralized authentication blockchainbased mechanism. In IEEE Wireless Communications and Networking Conf., pages 1-6, 2018

[280] Wei She et al. Blockchain Trust Model for Malicious Node Detection in Wireless Sensor Networks. IEEE Access, 7:38947 - 38956, 2019.

[281] M. Banerjee et al. Blockchain-Based Security Layer for Identification and Isolation of Malicious Things in IoT: A Conceptual Design. In 27th Int. Conf. on Computer Communication and Networks, pages 1-6. IEEE, 2018.

[282] V. Sharma. An Energy-Efficient Transaction Model for the Blockchainenabled Internet of Vehicles (IoV). IEEE Communications Letters, 23(2):246 - 249, 2018.

[283] A. Dorri et al. Blockchain: A distributed solution to automotive security and privacy. IEEE Communications Magazine, 55(12):119 - 125, 2017.

[284] Ali Dorri et al. Lsb: A lightweight scalable blockchain for iot security and privacy. arXiv preprint arXiv:1712.02969, pages 1-17, 2017.

[285] Ali Dorri et al. Blockchain for IoT security and privacy: The case study of a smart home. In IEEE Int. Conf. on Pervasive Computing and Communications Workshops (PerCom Workshops), pages 1-6. IEEE, 2017.

[286] Ali Dorri et al. Towards an optimized blockchain for IoT. In second Int. conf. on Internet-of-Things design and implementation, pages 173 178. IEEE, 2017.

[287] Z. Hong et al. A secure routing protocol with regional partitioned clustering and Beta trust management in smart home. Wireless Networks, 25:3805-3823, 2018.

[288] Mohammad Javad Amiri et al. Sharper: Sharding permissioned blockchains over network clusters. arXiv preprint arXiv:1910.00765, pages 1-25, 2019.

[289] Elli Androulaki et al. Hyperledger fabric: a distributed operating system for permissioned blockchains. In Proceedings of the thirteenth EuroSys conference, pages $1-15,2018$

[290] MA Rashid and Houshyar Honar Pajooh. A security framework for iot authentication and authorization based on blockchain technology. In 18th IEEE International Conference On Trust, Security And Privacy In Computing And Communications/13th IEEE International Conference On Big Data Science And Engineering (TrustCom/BigDataSE), pages 264-271. IEEE, 2019.

[291] Bacem Mbarek et al. Mbs: multilevel blockchain system for iot. Personal and Ubiquitous Computing, pages 1-8, 2019.

[292] Yogachandran Rahulamathavan et al. Privacy-preserving blockchain based IoT ecosystem using attribute-based encryption. In IEEE Int. Conf. on Advanced Networks and Telecommunications Systems (ANTS), pages 1-6. IEEE, 2017.

[293] Sidra Malik et al. ProductChain: Scalable Blockchain Framework to Support Provenance in Supply Chains. In IEEE 17th Int. Symposium on Network Computing and Applications (NCA), pages 1-10. IEEE, 2018

[294] Malavika Nair et al. The blockchain and increasing cooperative efficacy. Independent Review, 22(4):529-550, 2018.

[295] Loi Luu et al. Smartpool: Practical decentralized pooled mining. In 26th \{USENIX\} Security Symposium, pages 1409-1426, 2017.

[296] Ana Reyna et al. On blockchain and its integration with IoT. Challenges and opportunities. Future Generation Computer Systems, 88:173-190, 2018

[297] Xavier Chesterman. THE P2POOL MINING POOL, 2018.

[298] Open Networking Fundation. Software-defined networking: The new norm for networks. ONF White Paper, 2, 2012.

[299] N. McKeown et al. OpenFlow: enabling innovation in campus networks. ACM SIGCOMM Computer Communication Review, 38(2):6974, 2008

[300] NFVISG ETSI. Network functions virtualization, white paper, 2012.

[301] T. A. Al-Janabi et al. Efficient whale optimisation algorithm-based SDN clustering for IoT focused on node density. In 16th Annual Mediterranean Ad Hoc Networking Workshop (Med-Hoc-Net), pages 1-6. IEEE, 2017.

[302] Ahmed Abdelaziz et al. Distributed controller clustering in software defined networks. PloS one, 12(4):1-19, 2017.

[303] Hadar Sufiev et al. Dynamic SDN Controller Load Balancing. Future Internet, 11(3):1-21, 2019.

[304] Fatma Al Shuhaimi et al. Software defined network as solution to overcome security challenges in IoT. In 5th Int. Conf. on Reliability, Infocom Technologies and Optimization (Trends and Future Directions)(ICRITO), pages 491-496. IEEE, 2016.

[305] F. Olivier, G. Carlos, and N. Florent. Sdn based architecture for clustered wsn. In 2015 9th Int. Conf. on Innovative Mobile and Internet Services in Ubiquitous Computing, pages 342-347. IEEE, 2015. 
[306] Y. Nam et al. Clustered NFV service chaining optimization in mobile edge clouds. IEEE Communications Letters, 21(2):350 - 353, 2017.

[307] Jun Wu et al. NLES: A novel lifetime extension scheme for safetycritical cyber-physical systems using SDN and NFV. IEEE Internet of Things Journal, 6(2):2463 - 2475, 2018.

[308] A. Taherkordi and F. Eliassen. Scalable modeling of cloud-based iot services for smart cities. In 2016 IEEE International Conference on Pervasive Computing and Communication Workshops (PerCom Workshops), pages 1-6, 2016.

[309] Rustem Dautov et al. Data agility through clustered edge computing and stream processing. Concurrency and Computation: Practice and Experience, page e5093, 2018.

[310] J. Oueis et al. The fog balancing: Load distribution for small cell cloud computing. In IEEE 81st Vehicular Technology Conf., pages 1-6. IEEE, 2015.

[311] M. Elbamby et al. Proactive edge computing in latency-constrained fog networks. In European conference on networks and communications $(E u C N C)$, pages 1-6. IEEE, 2017.

[312] Xiaoqiang Shao et al. Dynamic IoT Device Clustering and Energy Management with Hybrid NOMA Systems. IEEE Transactions on Industrial Informatics, 14(10):4622 - 4630, 2018.

[313] R. Morabito et al. Evaluating Performance of Containerized IoT Services for Clustered Devices at the Network Edge. IEEE Internet of Things Journal, 4(4):1019-1030, 2017.

[314] Claus Pahl et al. A container-based edge cloud paas architecture based on raspberry pi clusters. In IEEE 4th Int. Conf. on Future Internet of Things and Cloud Workshops (FiCloudW), pages 117-124. IEEE, 2016.

[315] Shancang $\mathrm{Li}$ et al. A distributed consensus algorithm for decision making in service-oriented internet of things. IEEE Transactions on Industrial Informatics, 10(2):1461 - 1468, 2014.

[316] Leila Shooshtarian, Dapeng Lan, and Amir Taherkordi. A clusteringbased approach to efficient resource allocation in fog computing. In Pervasive Systems, Algorithms and Networks, pages 207-224. Springer, 2019.

[317] G. Akpakwu et al. A survey on 5G networks for the Internet of Things: Communication technologies and challenges. IEEE Access, 6:3619 3647, 2018.

[318] A. Gupta and R. K. Jha. A Survey of 5G Network: Architecture and Emerging Technologies. IEEE Access, 3:1206 - 1232, 2015.

[319] Shancang Li et al. 5G Internet of Things: A survey. Journal of Industrial Information Integration, 10(February):1-9, 2018.

[320] Waleed Ejaz et al. Internet of Things (IoT) in 5G wireless communications. IEEE Access, 4:10310 - 10314, 2016

[321] S K Goudos et al. A survey of IoT key enabling and future technologies: 5G, mobile IoT, sematic web and applications. Wireless Personal Communications, 97(2):1645-1675, 2017.

[322] Fadi Al-Turjman. Agile Medium Access in Smart-Cities Vehicular-IoT. In Smart Things and Femtocells. Taylor and Francis, 2018.

[323] Zhiguo Ding et al. A survey on non-orthogonal multiple access for 5G networks: Research challenges and future trends. IEEE Journal on Selected Areas in Communications, 35(10):2181 - 2195, 2017.

[324] S. Talwar et al. Enabling technologies and architectures for 5G wireless. In IEEE MTT-S Int. Microwave Symposium, pages 1-4. IEEE, 2014.

[325] A. Morgado et al. A survey of 5G technologies: Regulatory, standardization and industrial perspectives. Digital Communications and Networks, 4(2):87-97, 2018.

[326] I Chih-Lin et al. Toward green and soft: A 5G perspective. IEEE Communications Magazine, 52(2):66-73, 2014.

[327] C. Wang et al. Cellular architecture and key technologies for 5G wireless communication networks. IEEE communications magazine, 52(2): 122 - 130, 2014.

[328] S. A. Busari et al. Millimeter-wave massive MIMO communication for future wireless systems: A survey. IEEE Communications Surveys \& Tutorials, 20(2):836-869, 2018.

[329] Irfan Ahmed et al. A survey on hybrid beamforming techniques in 5G: Architecture and system model perspectives. IEEE Communications Surveys \& Tutorials, 20(4):3060 - 3097, 2018.

[330] X. Xia et al. A 5G-enabling technology: benefits, feasibility, and limitations of in-band full-duplex mMIMO. IEEE Vehicular Technology Magazine, 13(3):81 - 90, 2018.

[331] Rafay Iqbal Ansari et al. 5g d2d networks: Techniques, challenges, and future prospects. IEEE Systems Journal, 12(4):3970-3984, 2017.

[332] V. Jungnickel et al. The role of small cells, coordinated multipoint, and massive MIMO in 5G. IEEE Communications Magazine, 52(5):44 $-51,2014$.
[333] X. Wu et al. FlashLinQ: A synchronous distributed scheduler for peerto-peer ad hoc networks. IEEE/ACM Transactions on Networking, 21(4): $1215-1228,2013$.

[334] A. Kunz et al. Machine type communications in 3GPP: from release 10 to release 12. In IEEE Globecom Workshops, pages 1747-1752. IEEE, 2012.

[335] Shao-Yu Lien et al. Enhanced LTE device-to-device proximity services. IEEE Communications Magazine, 54(12):174 - 182, 2016.

[336] Anas Irfan and Aqeel Khalique. A Comparative Demonstration and Analysis of File Sharing Applications on Android Mobile Devices. International Journal of Computer Applications, 156(12):52-56, 2016.

[337] J. Harding et al. Vehicle-to-vehicle communications: readiness of $\mathrm{v} 2 \mathrm{v}$ technology for application. Technical report, United States. National Highway Traffic Safety Administration, 2014.

[338] Vincenzo Mancuso et al. Tie-breaking can maximize fairness without sacrificing throughput in D2D-assisted networks. In IEEE 17th Int. Symposium on A World of Wireless, Mobile and Multimedia Networks (WoWMoM), pages 1-9. IEEE, 2016.

[339] Selcuk Bassoy et al. Load aware self-organising user-centric dynamic CoMP clustering for 5G networks. IEEE Access, 4:2895 - 2906, 2016

[340] B. Romanous et al. Network densification: Challenges and opportunities in enabling 5g. In IEEE 20th International Workshop on Computer Aided Modelling and Design of Communication Links and Networks (CAMAD), pages 129-134. IEEE, 2015.

[341] Yingshuan Song et al. Intelligent access scheme for internet of things supported by $5 \mathrm{G}$ wireless network. Lecture Notes of the Institute for Computer Sciences, Social-Informatics and Telecommunications Engineering, LNICST, 237:341-351, 2018.

[342] L. Xu et al. A smart and balanced energy-efficient multihop clustering algorithm (smart-BEEM) for MIMO IoT systems in future networks. Sensors, 17(7):1-23, 2017.

[343] A. Asadi et al. Network-Assisted Outband D2D-Clustering in 5G Cellular Networks: Theory and Practice. IEEE Transactions on Mobile Computing, 16(8):2246 - 2259, 2017.

[344] X. Lin et al. The interplay between massive MIMO and underlaid D2D networking. IEEE Transactions on Wireless Communications, 14(6):3337 - 3351, 2015.

[345] Mahmoud Kamel et al. Ultra-dense networks: A survey. IEEE Communications Surveys \& Tutorials, 18(4):2522 - 2545, 2016. 